\title{
An Efficient Solution to the Factorized Geometrical Autofocus Problem
}

\author{
Jan Torgrimsson, Patrik Dammert, Senior Member, IEEE, Hans Hellsten, Member, IEEE, and \\ Lars M. H. Ulander, Senior Member, IEEE
}

\begin{abstract}
This paper describes a new search strategy within the scope of factorized geometrical autofocus (FGA) and syntheticaperture-radar processing. The FGA algorithm is a fast factorized back-projection formulation with six adjustable geometry parameters. By tuning the flight track step by step and maximizing focus quality by means of an object function, a sharp image is formed. We propose an efficient two-stage approach for the geometrical variation. The first stage is a low-order (few parameters) parallel search procedure involving small image areas. The second stage then combines the local hypotheses into one global autofocus solution, without the use of images. This method has been applied successfully on ultrawideband CARABAS II data. Errors due to a constant acceleration are superposed on the measured track prior to processing, giving a 6-D autofocus problem. Image results, including resolution, peak-to-sidelobe ratio and magnitude values for point-like targets, finally confirm the validity of the strategy. The results also verify the prediction that there are several satisfying autofocus solutions for the same radar data.
\end{abstract}

Index Terms-Autofocus, back-projection, synthetic aperture radar (SAR).

\section{INTRODUCTION}

$\mathbf{F}$ OR synthetic aperture radar (SAR), defocusing effects (phase errors) in the final image are primarily caused by geometrical errors or, essentially, GPS and inertial measurement unit (IMU) errors [4], [17].

Slow deviations (i.e., on the scale of integration time) from a linear flight track (antenna phase center) must be measured within fractions $(\sim 1 / 16)$ of the center wavelength $\lambda_{c}$ to avoid image artifacts [4], [17]. Fast fluctuations give other criteria [4]. For high radar bands (e.g., X-band) and extended tracks (apertures), $\lambda_{c} / 16$ accuracy is typically too strict to form full-resolution imagery. Even for a low band such as very high frequency (VHF), issues such as IMU drift and/or a jammed/shadowed GPS will degrade the image quality [26]-[28].

Manuscript received May 19, 2015; revised December 18, 2015; accepted March 1, 2016. Date of publication April 25, 2016; date of current version June 1, 2016. This work was supported by the Swedish Governmental Agency for Innovation Systems (VINNOVA).

J. Torgrimsson is with Chalmers University of Technology, Gothenburg 412 58, Sweden (e-mail: jan.torgrimsson@chalmers.se).

P. Dammert and H. Hellsten are with SAAB Electronic Defence Systems, Gothenburg 412 89, Sweden (e-mail: patrik.dammert@saabgroup.com; hans. hellsten@saabgroup.com).

L. M. H. Ulander is with Swedish Defence Research Agency (FOI), Stockholm 164 90, Sweden, and also with Chalmers University of Technology, Göthenburg 412 58, Sweden (e-mail: lars.ulander@foi.se).

Digital Object Identifier 10.1109/TGRS.2016.2550663
Autofocus algorithms are usually utilized to compensate for an erroneous geometry (or for inadequate motion compensation). In the traditional sense, information in a defocused image is used to estimate and correct space invariant 1-D phase errors (in azimuth) [4], [17].

Standard techniques include mapdrift (MD) [4], [17] and the phase gradient algorithm (PGA) [4], [6], [7], [16], [17]. These schemes are well known and often used in practice. After the estimation procedure, phase errors are canceled via a complex multiplication.

Metric-based techniques, e.g., [1], [11], [19], [22], [24], and [32], have also received a fair deal of attention. These schemes can potentially produce a better result than MD and the PGA, i.e., at an acceptable run time. In principle, the complex multiplication is repeated with different compensation factors until an object function is optimized.

However, techniques relying on the constrained phase error model (see above) are gradually becoming performance limited. This is due to a trend leaning toward finer and finer resolution, together with a desire to relax requirements on the GPS/IMU (to reduce costs and relieve export restrictions). With this in mind, a number of more advanced autofocus algorithms have been developed.

The 2-D PGA [12] and different PGA [5]/PGA-MD [33] extensions can correct 2-D phase errors or residual range cell migration (RCM).

Block processing may in turn mitigate space-variant defocusing effects [4], [17], [33]. Note that stripmap routines partially alleviate these effect in azimuth as phase errors for adjacent apertures are estimated [11], [33].

Another class of autofocus algorithms tries to extract geometrical parameters in various ways. These are then employed in the processing to form a focused image. Early ideas are discussed in [18] and [21]. Recent schemes based on MD and the PGA are described in [20] and [31]. Multilateration methods based on prominent point phase tracking [2] and local 2-D MD [3] should also be mentioned in this context. This class increases the capability but does not provide a full geometrical solution.

Complete compensation of an erroneous geometry calls for a time-domain algorithm [29]. Lately, a novel geometrical autofocus approach has been introduced, i.e., factorized geometrical autofocus (FGA) [26], [27]. This approach provides a full geometrical solution [26], [27].

The FGA algorithm is integrated in a fast factorized backprojection (FFBP) [29] chain. A base-two factorization (i.e., this 
FGA formulation is restricted to base two as opposed to FFBP in general) relying on geometry regulation is executed in a horizontal plane [26], [27]. Six independent parameters are adopted for each subaperture pair; these are adjusted until a sharp image is obtained (for a given pair, at the factorization step in question), and an object function grades the focus quality [26], [27].

The geometry is varied step by step via triangles in 3-D space, a course of action that can correct residual space-variant RCM. However, thus far, tests on real SAR data have only demonstrated this for a constrained 1-D autofocus problem [26].

The FGA algorithm is a high-performance technique; searching the parameter space is however a challenging task, restricting its practical use. This issue has only been covered partially thus far. In [27], a sensitivity analysis is described, which is developed to compare two different geometry hypotheses. This tool can be employed to decide if all six quantities are indispensable (again, for a given subaperture pair, at the factorization step in question) or if a subset will suffice, i.e., from a focus perspective. The same reference seeks to find an optimal geometry hypothesis by performing a Broyden-Fletcher-GoldfarbShanno (BFGS) [23] search in parameter space to minimize the object function. However, the conclusion is that there is a major risk of getting trapped in a shallow local minimum as the function is not convex [27].

The objective of this paper is to formulate a robust search strategy and test it on real data, i.e., within the scope of the FGA algorithm and a 6-D autofocus problem.

A natural extension is to let the BFGS search follow a sparse grid search, to coarse-tune the geometry first. However, in higher dimensions, this method is time-consuming. In addition, the full image will not necessarily give a satisfying solution.

We propose a two-stage approach, made up of a block search and a local-to-global computation. The first stage tries to avoid local minima by performing a low-order (one parameter if feasible) search on small image areas. The second stage continues by using the sensitivity analysis to fit a geometry for the full image. The aim is to find a global solution based on the block search. In essence, the local-to-global computation is time efficient; as there is no need for image formation, basic trigonometry does the job.

The FGA algorithm with the new search strategy incorporated, has been applied in spotlight mode on ultrawideband VHF data, i.e., on real Coherent All RAdio BAnd System II (CARABAS II) [14] data. Errors due to a constant acceleration are superposed on the measured flight track prior to processing, to simulate a 3-D drifting IMU (without GPS support). This defines a 6-D problem, implying that all six parameters are erroneous, but not necessarily defocusing.

In this paper, resulting FGA images will be presented, analyzed, and compared to focused reference images (no errors superposed on the measured flight track prior to processing) and to defocused images formed without autofocus, in consequence suffering from residual space-variant RCM.

However, before actually satisfying the stated objective, a review is required, dealing with time-domain SAR processing, the FGA concept, BFGS, the sensitivity analysis, and of course, with the new search strategy. The realization and evaluation procedure will be recapped in detail as well.

\section{PReliminaries}

\section{A. Time-Domain SAR Processing}

Global back-projection (GBP) [29] is a time-domain algorithm, projecting pulse compressed radar echoes to a focus target plane (FTP) and on to a generally defined image. Each sample position along track contributes with a data value $D$ to each pixel. Complex values are added coherently, causing interference, resolving reflective structures.

The polar GBP expression is as follows:

$$
I(\rho, \theta)=\sum_{n=1}^{N} D\left(n, R_{n}\right) \cdot R_{n} \cdot \exp \left(\frac{j 4 \pi R_{n}}{\lambda_{d}}\right) .
$$

In (1), $I$ is an image formed from data acquired along an arbitrary flight track, extending across $N$ sample positions (for simplicity, presume that $N$ equals a power of two). The pixel coordinate $(\rho, \theta)$ originates from the center of a user-defined aperture vector and hits a point in the FTP. The slant range $R_{n}$ between the sample position (with index $n$ ) and the point in question (corresponding to a coordinate) determines which data value $D\left(n, R_{n}\right)$ to accumulate; the range interpolation retrieves the proper value from available samples. For demodulated data, the value must also be multiplied by a phase factor, i.e., the exponential. $\lambda_{d}$ is the demodulation wavelength. The range multiplication is included to establish $1 / R$ dependence [29]. Note also that this formulation omits a ramp filter, usually applied to calibrate the spectral support [29]. This can be viewed as a postprocessing step.

GBP is indeed a versatile algorithm; however, its use is normally restricted to moderately sized images due to the number of operations involved (proportional to $N^{3}$ for $N$ sample positions and an $N \times N$ image) [29].

FFBP [29] is a time-saving alternative, combining pulse compressed radar echoes coherently step by step.

Consider (1) and the consequence of dividing the sum in (1) into two terms, i.e.,

$$
\begin{aligned}
I(\rho, \theta)= & \sum_{n=1}^{N / 2} D\left(n, R_{n}\right) \cdot R_{n} \cdot \exp \left(\frac{j 4 \pi R_{n}}{\lambda_{d}}\right) \\
& +\sum_{n=N / 2+1}^{N} D\left(n, R_{n}\right) \cdot R_{n} \cdot \exp \left(\frac{j 4 \pi R_{n}}{\lambda_{d}}\right) .
\end{aligned}
$$

The terms in (2) may be viewed as two low-resolution realizations of $I$. In principle, the aperture (the track) is partitioned into subapertures; hence, the azimuth bandwidth is halved (one half for each subaperture). Therefore, subimages can be formed with a reduced sampling rate (approximately half the original rate), and the terms can in turn be attained by way of subimage interpolation.

The polar base-two FFBP expression is as follows:

$$
\begin{aligned}
I(\rho, \theta)=I_{1}\left(\rho_{1}, \theta_{1}\right) \cdot \exp \left(\frac{j 4 \pi \Delta \rho_{1}}{\lambda_{d}}\right) & \\
+ & I_{2}\left(\rho_{2}, \theta_{2}\right) \cdot \exp \left(\frac{j 4 \pi \Delta \rho_{2}}{\lambda_{d}}\right) .
\end{aligned}
$$


In (3), $I$ is formed by adding a contribution from subimage $I_{1}$ and $I_{2}$ (corresponding to the terms). To elucidate, the pixel coordinate $(\rho, \theta)$ originates from the center of a user-defined aperture vector and hits a point in the FTP. Subimage coordinates $\left(\rho_{1}, \theta_{1}\right)$ and $\left(\rho_{2}, \theta_{2}\right)$ emerge from the subapertures (in practice, from user-defined subaperture vectors) and converge at this point. Data values $I_{1}\left(\rho_{1}, \theta_{1}\right)$ and $I_{2}\left(\rho_{2}, \theta_{2}\right)$ are determined by the coordinates; interpolation (in both range and angle [10], [29]) retrieves proper values from available samples. As in (1), exponentials (with $\Delta \rho_{1}=\rho-\rho_{1}$ and $\Delta \rho_{2}=\rho-\rho_{2}$ ) compensate for demodulation (note that the phase factors in (3) are only valid for slant-based images).

The sum in (1) can be divided into $N / 2$ factorization terms, a multistep $\left(\log _{2} N\right)$ approach may then be employed to obtain $I$. The principle is the same as above: the aperture (the track) is partitioned into subapertures, increasing in length (finer angular resolution) and decreasing in number for each factorization step [29]. Subapertures come with subimages, i.e., with coordinates in range and sublobe angle. The antenna beam is split up into sublobes.

Ideally, if the number of sample positions can be expressed as a factorization of integers, the final step gives the polar aperture image $I$.

The number of operations for a base-two implementation is proportional to $2 N^{2} \log _{2} N$ (again for $N$ sample positions and an $N \times N$ image), i.e., under the condition that $N$ equals a power of two [29]. Image quality requirements may however motivate a less effective algorithm execution (e.g., by reducing the number of steps and/or using a better interpolator), to make up for the fact that interpolation errors are accumulated for each factorization step [10], [29].

\section{B. $F G A$}

The FGA concept [26], [27] will now be described in detail; the text concentrates on the autofocus function, but applies to conventional FFBP processing (without autofocus) as well. Customary SAR premises, e.g., the start-stop and the Born approximation, and a constant wave velocity are assumed to be valid [29].

To set the tone, presume that pulse compressed radar echoes are demodulated and factorized with base two until two subapertures remain (see Fig. 1). The image and the FTP coincide with the horizontal plane, i.e., the $x y$ plane.

Despite measurement errors, the subimages are focused. This is due to limited angular resolution. The aperture $\left(\boldsymbol{Q}_{13}\right)$ is synthesized as a vector (aligned with the nominal flight direction), extending from the start point $\left(p_{1}\right)$ of the first subaperture $\left(Q_{12}\right)$ to the end point $\left(p_{3}\right)$ of the other $\left(Q_{23}\right) . p_{1}, p_{3}$, and the cutoff point $\left(p_{2}\right)$ between $Q_{12}$ and $Q_{23}$ form a triangle (see Fig. 2) or a line as a special case. If the geometry is too erroneous (due to measurement errors), the aperture image will be defocused.

By varying parameters defining the triangle, different geometry hypotheses can be assessed [26], [27]. Confining the variation (the fewer parameters, the better) is of utmost importance. The geometry may be described by means of an altitude, three

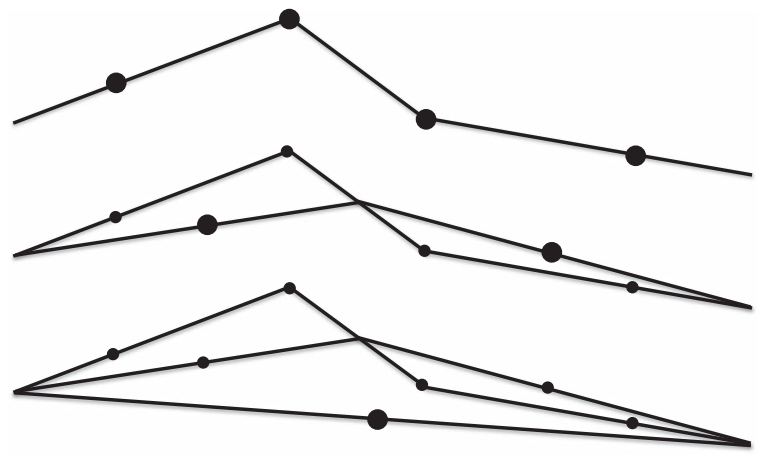

Fig. 1. (Top) Four sample positions (tied together). (Middle) After the first factorization step, two subapertures (and two new positions) remain. (Bottom) The second and final factorization step gives the aperture (and one new position).

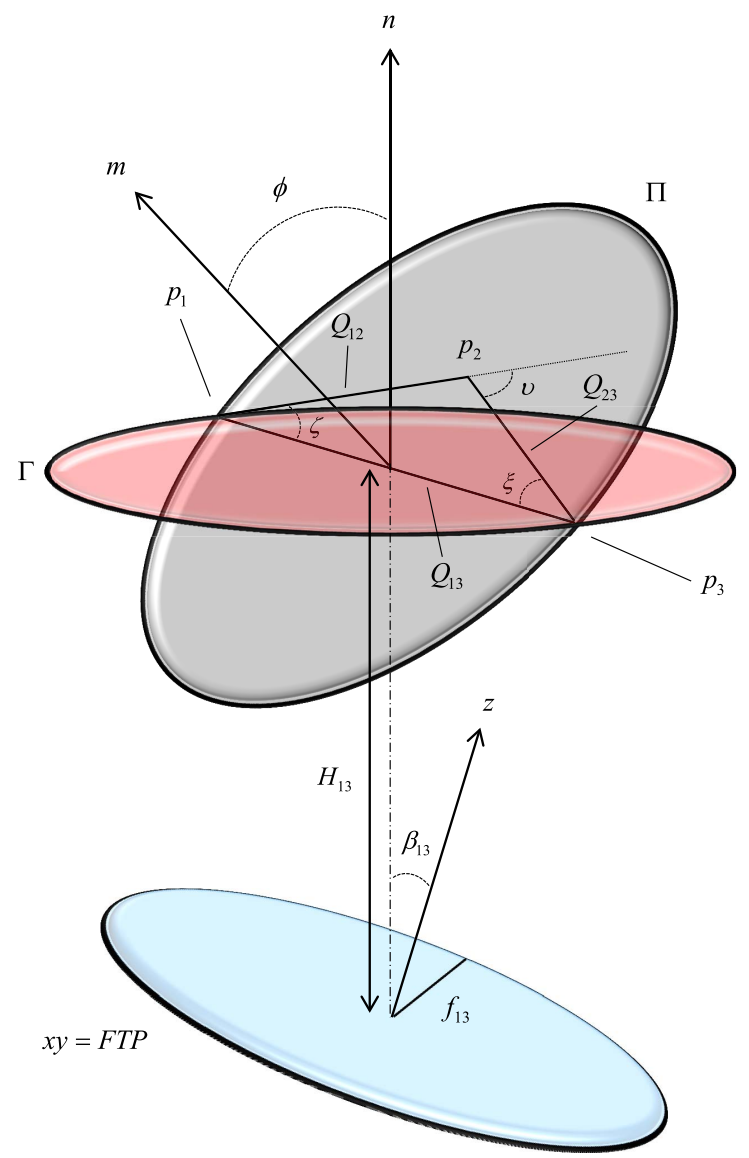

Fig. 2. Triangle in the plane $\Pi$ (gray) with a normal vector $m . v, \phi, \beta_{13}, H_{13}$, $\zeta$, and $\xi$ are essential parameters in this autofocus formulation. Note that $Q_{13}$ and a vector in the $x y$-plane (blue), orthogonal to $Q_{\mathbf{1 3}}\left(\boldsymbol{f}_{\mathbf{1 3}}\right)$ define the plane $\Gamma$ (red) with a normal vector $n . x y z$ is a local Cartesian coordinate system. In practice, the $x y$ plane is horizontal with a vertical $z$. The geometry given has been tilted for clarity.

angles, and two length variables, all in all six independent parameters [26], [27], chosen as follows (see Fig. 2):

- $v$ : angle between $Q_{12}$ and $Q_{23}$;

- $L_{13}$ : length of $\boldsymbol{Q}_{\mathbf{1 3}}$;

- $\Delta L$ : length difference between $Q_{12}$ and $Q_{23}$;

- $\phi$ : angle between $\boldsymbol{\Gamma}$ and $\boldsymbol{\Pi}$;

- $\beta_{13}$ : angle between the $x y$ plane and $Q_{13}$;

- $H_{13}$ : altitude at the center of $\boldsymbol{Q}_{13}$. 


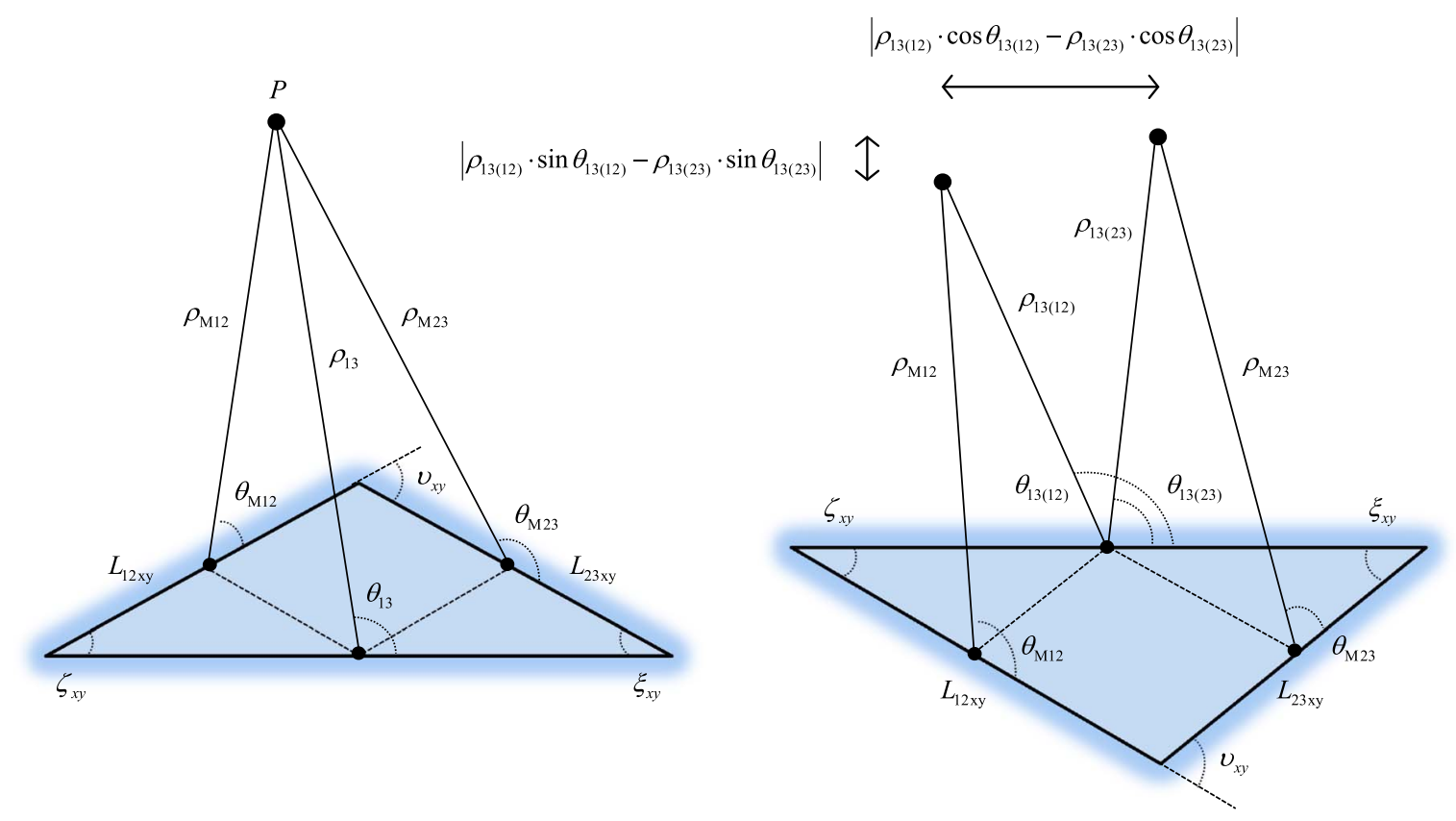

Fig. 3. (Left) 2-D geometry for the M transform (i.e., the triangle in Fig. 2 projected to the $x y$ plane). Point $P$ is an arbitrary point in the image display plane or FTP. In practice, $P$ and the vertex of the cutoff point can be located on either side of the aperture, explaining the signs in (4)-(7). (Right) A case where the geometry hypothesis has been altered prior to inverse transformation, giving different coordinate sets and hence a shift. In processing terms, this implies that erroneous data values will be attained when interpolating, possibly producing a defocused image depending on the magnitude of the shift.

The variation is carried out consecutively by a merging $(\mathrm{M})$ and a range history preserving (RHP) transform.

In principle, an arbitrary geometry hypothesis is expressed in the chosen parameters. Supporting quantities are computed, and the pixel coordinates $\left(\rho_{13}, \theta_{13}\right)$ of the aperture image $\left(I_{13}\right)$ are established.

The $\mathrm{M}$ transform then converts these to corresponding subimage coordinates, i.e., giving $\left(\rho_{M 12}, \theta_{M 12}\right)$ and $\left(\rho_{M 23}, \theta_{M 23}\right)$.

This is equivalent to translating and rotating subaperture vectors horizontally (as opposed to translating and rotating an intact triangle). Fig. 3 (Left) and the following equations clarify the idea behind the $\mathrm{M}$ transform:

$\rho_{M 12}=\left(\rho_{13}^{2}+\left(\frac{L_{23 x y}}{2}\right)^{2}-\rho_{13} \cdot L_{23 x y} \cdot \cos \left(\pi-\theta_{13} \pm \xi_{x y}\right)\right)^{\frac{1}{2}}$

$\theta_{M 12}=\cos ^{-1}\left(\frac{\rho_{13}^{2}-\rho_{M 12}^{2}-\left(\frac{L_{23 x y}}{2}\right)^{2}}{-\rho_{M 12} \cdot L_{23 x y}}\right) \pm v_{x y}$

$\rho_{M 23}=\left(\rho_{13}^{2}+\left(\frac{L_{12 x y}}{2}\right)^{2}-\rho_{13} \cdot L_{12 x y} \cdot \cos \left(\theta_{13} \pm \zeta_{x y}\right)\right)^{\frac{1}{2}}$

$\theta_{M 23}=\pi-\cos ^{-1}\left(\frac{\rho_{13}^{2}-\rho_{M 23}^{2}-\left(\frac{L_{12 x y}}{2}\right)^{2}}{-\rho_{M 23} \cdot L_{12 x y}}\right) \pm v_{x y}$.

where $L_{12 x y}$ and $L_{23 x y}$ are the horizontal length variables (associated with the subapertures), whereas $v_{x y}, \zeta_{x y}$, and $\xi_{x y}$ are projected angles $(v, \zeta$, and $\xi)$.
M-transformed coordinates are distorted by the RHP transform, i.e., giving $\left(\rho_{M 12}^{\prime}, \theta_{M 12}^{\prime}\right)$ and $\left(\rho_{M 23}^{\prime}, \theta_{M 23}^{\prime}\right)$. This is equivalent to prolonging/shortening, tilting, and translating subaperture vectors vertically. Note that the first two items also impact the $\mathrm{M}$ transform. The following equations clarify the idea behind the RHP transform (with subscripts dropped, i.e., either 12 or 23 ):

$$
\begin{aligned}
\rho_{M}^{\prime} & =\sqrt{\left(\rho_{M}^{2}+H^{2}-H_{0}^{2}\right)} \\
\theta_{M}^{\prime} & =\cos ^{-1}\left(\frac{\rho_{M} \cdot L_{x y} \cdot \cos \theta_{M}-H \cdot L_{z}+H_{0} \cdot L_{0 z}}{\rho_{M}^{\prime} \cdot L_{0 x y}}\right) .
\end{aligned}
$$

Note that $\rho_{M}$ is the range, and $\theta_{M}$ is the sublobe angle (in the $x y$ plane). $H_{0}$ is the altitude, defined at the center of the subaperture, same as $\rho_{M}$ and $\theta_{M} . L_{0 x y}$ is the horizontal length, whereas $L_{0 z}$ is the vertical length (signed). The subscript zero signifies that $H_{0}, L_{0 x y}$ and $L_{0 z}$ are specified by means of an initial geometry hypothesis, derived from the measured flight track. $H, L_{x y}$, and $L_{z}$ in turn correspond to an arbitrary hypothesis.

Each subimage is interpolated to M- and RHP-transformed coordinates (corresponding to the arbitrary geometry hypothesis). A coherent addition then gives the aperture image as follows (note that phase factors are omitted):

$$
I_{13}\left(\rho_{13}, \theta_{13}\right)=I_{12}\left(\rho_{M 12}^{\prime}, \theta_{M 12}^{\prime}\right)+I_{23}\left(\rho_{M 23}^{\prime}, \theta_{M 23}^{\prime}\right) \text {. }
$$

Fundamentally, the final factorization step is repeated time after time. Each hypothesis yields an image. To decide if the focus quality is satisfying, the similarity between the terms in (10) is calculated by way of intensity correlation. If the normalized correlation sum is adequately close to unity, the output of the object function $F$ in (11) goes low, and the aperture image 
is autofocused. If not, another geometry hypothesis must be assessed.

Ordinary (FFBP) processing on the contrary adopts the initial hypothesis. Note also that, in this case, the RHP transform is redundant, as it basically becomes a unity transform.

In the following equation, $g=\left|I\left(\rho_{M}^{\prime}, \theta_{M}^{\prime}\right)\right|^{2}$, and $m$ is the corresponding average value (subscripts dropped):

$F=1-\frac{\sum \sum\left(g_{12}-m_{12}\right) \cdot\left(g_{23}-m_{23}\right)}{\sqrt{\left(\sum \sum\left(g_{12}-m_{12}\right)^{2}\right) \cdot\left(\sum \sum\left(g_{23}-m_{23}\right)^{2}\right)}}$.

The summation is over all pixels.

Before proceeding, it should be emphasized that although one FGA step (i.e., a factorization step with adjustable parameters) is presumed here, the autofocus function can be activated at any time during the factorization [26], [27]. However, as the need for measurement accuracy increases in a quadratic fashion with subaperture length, it is more likely that the algorithm is required later on in the FFBP chain.

\section{C. $B F G S$}

The BFGS method [23] is an iterative algorithm, employed to solve unconstrained, nonlinear optimization problems in multiple variables (i.e., solving for extrema or zeroes).

The routine approximates Newton's method by estimating the Hessian via the gradient of the object function (deduced during the search). This approach has the potential to converge at a superlinear rate.

The expression for a BFGS iteration is as follows:

$$
\boldsymbol{u}_{k+1}=\boldsymbol{u}_{k}-\mu_{k} \boldsymbol{B}\left(\boldsymbol{u}_{k}\right)^{-1} \nabla \boldsymbol{F}\left(\boldsymbol{u}_{k}\right) .
$$

The six independent parameters (or a subset of these) are retained in $\boldsymbol{u} . k+1$ is the current iteration, whereas $k$ is the previous. $\mu$ is the size of the search step, whereas $\boldsymbol{B}$ in turn is the estimated Hessian (note that in Newton's method, $\boldsymbol{B}$ is the true Hessian, and in the gradient (steepest) descent method, $\boldsymbol{B}$ is an identity matrix [23]). $\nabla \boldsymbol{F}$ is ultimately the gradient of a general object function.

\section{METHOD}

\section{A. Sensitivity Analysis}

As the sensitivity analysis [27] plays a key part in context of the new search strategy, it will be introduced first.

1) Algorithm: It is evident, that if M- and RHP-transformed coordinates are inserted into an Inverse RHP transform (IRHP), as shown in the following equations, M-transformed coordinates will be the result, i.e., $\left(\rho_{M 12}, \theta_{M 12}\right)$ and $\left(\rho_{M 23}, \theta_{M 23}\right)$ :

$$
\begin{aligned}
\rho_{M} & =\sqrt{\left(\rho_{M}^{\prime 2}-H^{2}+H_{0}^{2}\right)} \\
\theta_{M} & =\cos ^{-1}\left(\frac{\rho_{M}^{\prime} \cdot L_{0 x y} \cdot \cos \theta_{M}^{\prime}+H \cdot L_{z}-H_{0} \cdot L_{0 z}}{\rho_{M} \cdot L_{x y}}\right) .
\end{aligned}
$$

It is also evident, that if M-transformed coordinates are inserted into an Inverse M transform (IM), as shown in the following equations, original pixel coordinates (with subscripts, i.e., either 12 or 23$)$ will be the result, i.e., $\left(\rho_{13(12)}, \theta_{13(12)}\right)$ and $\left(\rho_{13(23)}, \theta_{13(23)}\right)$ :

$$
\begin{gathered}
\rho_{13(12)=}\left(\cos \left(\theta_{M 12} \pm v_{x y}\right) \cdot\left(-\rho_{M 12} \cdot L_{23 x y}\right)\right. \\
\left.\quad+\rho_{M 12}^{2}+\left(\frac{L_{23 x y}}{2}\right)^{2}\right)^{\frac{1}{2}} \\
\theta_{13(12)}=\pi-\cos ^{-1}\left(\frac{\rho_{M 12}^{2}-\rho_{13(12)}^{2}-\left(\frac{L_{23 x y}}{2}\right)^{2}}{-\rho_{13(12)} \cdot L_{23 x y}}\right) \pm \xi_{x y}
\end{gathered}
$$

$$
\begin{aligned}
& \rho_{13(23)}=\left(\cos \left(\pi \pm v_{x y}-\theta_{M 23}\right) \cdot\left(-\rho_{M 23} \cdot L_{12 x y}\right)\right. \\
&\left.+\rho_{M 23}^{2}+\left(\frac{L_{12 x y}}{2}\right)^{2}\right)^{\frac{1}{2}} \\
& \theta_{13(23)}=\cos ^{-1}\left(\frac{\rho_{M 23}^{2}-\rho_{13(23)}^{2}-\left(\frac{L_{12 x y}}{2}\right)^{2}}{-\rho_{13(23)} \cdot L_{12 x y}}\right) \pm \zeta_{x y} .
\end{aligned}
$$

These two sets will of course be identical, as long as the geometry hypothesis is the same in the forward, and in the inverse transforms.

Now, if the hypothesis is altered prior to inverse transformation (IRHP and IM), the sets will not necessarily be identical [see Fig. 3 (Right)]. The shift between corresponding coordinates (in the two sets) can be computed in azimuth and range. By utilizing the forward and inverse transforms like this, relations between two arbitrary geometries may be studied, i.e., sensitivity analysis [27].

Logically, the measured track represents the first geometry, and the second is the first with (expected) measurement errors superposed. Fundamentally, the shift gives a direct indication of the focus level in an aperture image or in a subimage, formed with an erroneous geometry.

In the following equations, the normalized absolute shift is calculated in azimuth and range, respectively, (normalized by half a resolution cell $\left(\delta_{\alpha}\right)$ and by $\left.\lambda_{c} / 16\right)$ :

$$
\begin{aligned}
\varepsilon_{\alpha} & =\frac{\left|\rho_{13(12)} \cdot \cos \theta_{13(12)}-\rho_{13(23)} \cdot \cos \theta_{13(23)}\right|}{\left(\frac{\delta_{\alpha}}{2}\right)} \\
\varepsilon_{\rho} & =\frac{\left|\rho_{13(12)}-\rho_{13(23)}\right|}{\left(\frac{\lambda_{c}}{16}\right)} .
\end{aligned}
$$

If either $\varepsilon_{\alpha}$ or $\varepsilon_{\rho}$ exceeds unity (the focus criteria), the aperture image (or the subimage) will be defocused. Note that, in [27], $\varepsilon_{\rho}$ is normalized by half a resolution cell in range. This definition is however not strict enough in general (as it yields an incoherent criterion).

2) Applications: The sensitivity analysis can be used to determine the number of autofocus or FGA steps required, i.e., for a given (expected) error scenario. This is achieved by producing $\varepsilon_{\alpha^{-}}$and $\varepsilon_{\rho}$-plots for coordinates of interest, up to the last factorization step where all values are below unity (for 


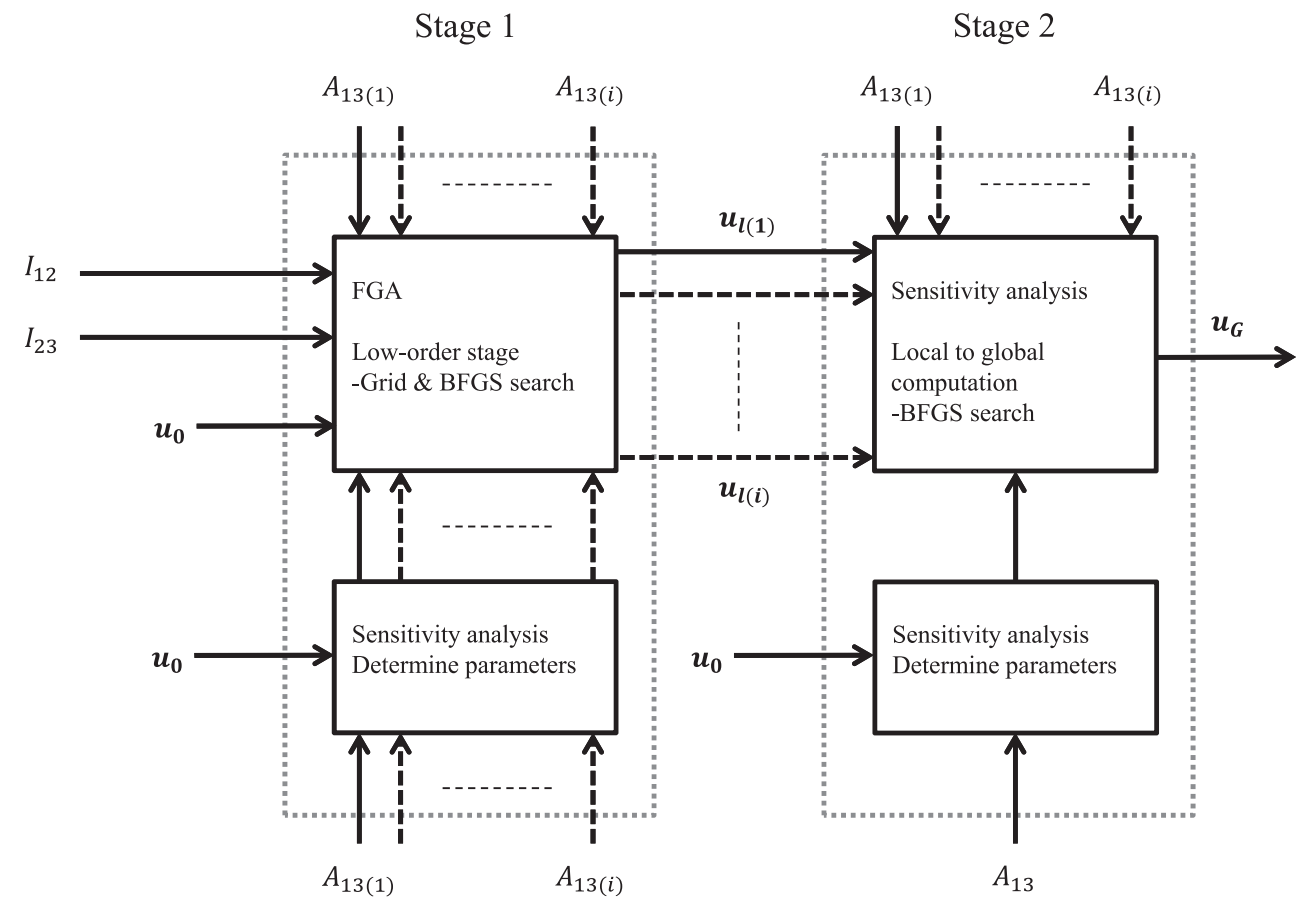

Fig. 4. Flowchart for the new search strategy. $A_{13(1)}$ to $A_{13(i)}$ are image areas (i.e., coordinates for each block in the image to be autofocused). $\boldsymbol{u}_{\mathbf{0}}$ is in turn the initial geometry hypothesis, $\boldsymbol{u}_{\boldsymbol{l}(\mathbf{1})}$ to $\boldsymbol{u}_{\boldsymbol{l}(\boldsymbol{i})}$ are the low-order solutions (hypotheses) for $i$ areas, and $\boldsymbol{u}_{\boldsymbol{G}}$ is the global solution. Note that the low-order stage has a completely parallel structure, i.e., a simultaneous search for $i$ hypotheses. The comparison of $\boldsymbol{u}_{\boldsymbol{l}(\mathbf{1})}$ to $\boldsymbol{u}_{\boldsymbol{l}(\boldsymbol{i})}$ with $\boldsymbol{u}_{\boldsymbol{G}}$ is also parallel, implying implementation efficiency.

all subimages at that step). At this step, the autofocus function must be activated to eventually recover a focused image.

The sensitivity analysis can in addition be used to determine the necessary quantities at each FGA step. This is achieved by adjusting parameters (a subset) for the second geometry (the hypothesis with measurement errors superposed) while comparing it to the first (the measured track). Various parameter combinations (the number of parameters, which parameters and finally their values should be considered) are tried out again until all values in the $\varepsilon_{\alpha}$-and $\varepsilon_{\rho}$-plots are satisfying (below unity for all subimages at the step in question). This is an optimization problem that can be solved by means of a BFGS search in the limited parameter space, i.e., minimization with respect to maxima in the plots.

\section{B. New Search Strategy}

In previous FGA tests, quite basic search strategies have been employed.

In [26], a 1-D grid search is adopted for a constrained autofocus problem (synthetic and real data); this routine is robust, but very slow, as each grid point (geometry hypothesis) calls for image formation (interpolation). In consequence, an exhaustive 6-D search is not a feasible approach in practice, even a 3-D search is an overwhelming task.

In [27], a direct BFGS search is adopted for a 6-D autofocus problem (synthetic data); this routine is rather fast but requires a very smooth object function to perform well. Accurate interpolation may in turn slow the search down substantially (naturally, this is true for a grid search as well). This is unfortunate, as a good interpolator in general gives a smoother function.
TABLE I

System And Geometry QuANTities. The IMAges Shown ARE MiNOR CUTS OF IMAGES Formed FOR THE SCENE SIZE TABUlated Below. The SAMPle SPACING (GROUND RANGE AND COSINE OF ANGLE) IS REPORTED FOR IMAGE DATA AND THE SQUinT ANGLE $\left(0^{\circ}\right)$ IMPLIES BROADSIDE IMAGING. NOTE THAT THE Range SPacing and the Altitude Differ Between the First Data Set (VIDSEL) AND THE SECOND (LINKÖPING)

\begin{tabular}{lr}
\hline Quantity & Value \\
\hline Centre frequency & $55 \mathrm{MHz}$ \\
Bandwidth & $70 \mathrm{MHz}$ \\
Ground range spacing & $0.52 / 0.49 \mathrm{~m}$ \\
Cosine of angle spacing & $8.63 \cdot 10^{-5}$ \\
Integration angle & $45^{\circ}-60^{\circ}$ \\
Squint angle & $0^{\circ}$ \\
Range (scene centre) & $16000 \mathrm{~m}$ \\
Size of scene & $15400 \times 6100 \mathrm{~m}$ \\
Aperture length & $15400 \mathrm{~m}$ \\
Altitude & $6400 / 4500 \mathrm{~m}$ \\
\hline
\end{tabular}

The new search strategy is a two-stage approach, including both methods mentioned earlier (i.e., to exploit the advantages). This section and the flowchart in Fig. 4 provide a detailed formulation.

1) Stage 1: The aperture image, or subimage to be autofocused, is first divided into small areas (blocks). This is a tradeoff situation. In support of the sensitivity analysis, the chosen size should confine the number of parameters for the block search, ideally to a single quantity. In addition, the areas must be adequately large to give reliable results, but preferably also 

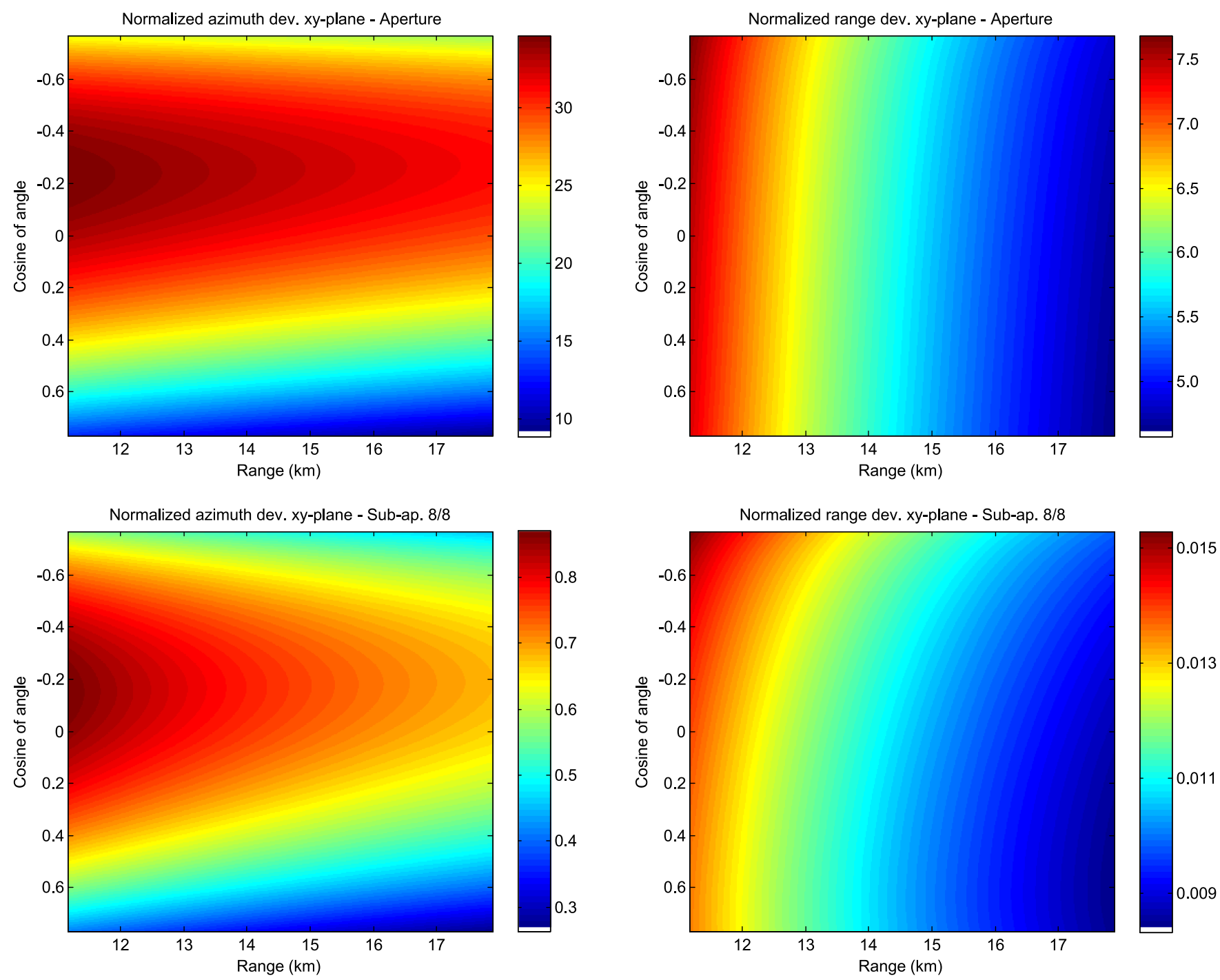

Fig. 5. Sensitivity plots for the first data set (Vidsel), corresponding to a scenario where an erroneous $x y z$-acceleration $\left(a_{x}=+0.0025 \mathrm{~m} / \mathrm{s}^{2}, a_{y}=\right.$ $-0.0025 \mathrm{~m} / \mathrm{s}^{2}$, and $a_{z}=+0.0025 \mathrm{~m} / \mathrm{s}^{2}$ ) has been superposed on the measured flight track. (Top, left) $\varepsilon_{\alpha}$-plot for the final factorization step. (Top, right) $\varepsilon_{\rho}$-plot for the final factorization step. (Bottom, left to right) Worst-case sensitivity plots, three factorization steps back. Note that the top plots have values well above unity, whereas the bottom plots have values below.

numerous, to assure that the second stage is not affected by a couple of poor solutions.

A low-order search is performed for each image area. This procedure is parallel in structure (see Fig. 4). A sparse grid is examined for a suitable point. The BFGS search is then initiated (from that point) to fine-tune the geometry, i.e., to find a better solution. By limiting the parameter space and coarse-tuning the geometry to begin with, local minima are avoided.

The ambition is now to fit a geometry for the full image (comprising all areas). Contrary to the first stage, the second stage does not utilize the FGA algorithm. A local-to-global computation is used instead.

2) Stage 2: The sensitivity analysis is used to determine the necessary quantities to autofocus the full image.
Low-order solutions are then compared to a global geometry hypothesis, once again by means of the analysis. The shift is computed in two orthogonal dimensions [see Fig. 3 (Right)] and the norm is taken, i.e., for the center coordinate of each image area. This comparison is parallel in structure as well (see Fig. 4).

The sum in (21), shown at the bottom of the page, is a direct measure of equality between individual solutions and the proposed global hypothesis.

The BFGS method is employed to minimize the object function $\left(F_{l 2 g}\right)$ in (21) (as opposed to the preceding minimization of $F$ ). The local-to-global computation is time efficient; as no images are formed, trigonometry is all that matters. Essentially, $F_{l 2 g}$ is based on elementary mathematical functions; images are laid aside after the first stage of the strategy.

$$
F_{l 2 g}=\sum \sqrt{\left(\rho_{13(12)} \cdot \cos \theta_{13(12)}-\rho_{13(23)} \cdot \cos \theta_{13(23)}\right)^{2}+\left(\rho_{13(12)} \cdot \sin \theta_{13(12)}-\rho_{13(23)} \cdot \sin \theta_{13(23)}\right)^{2}}
$$



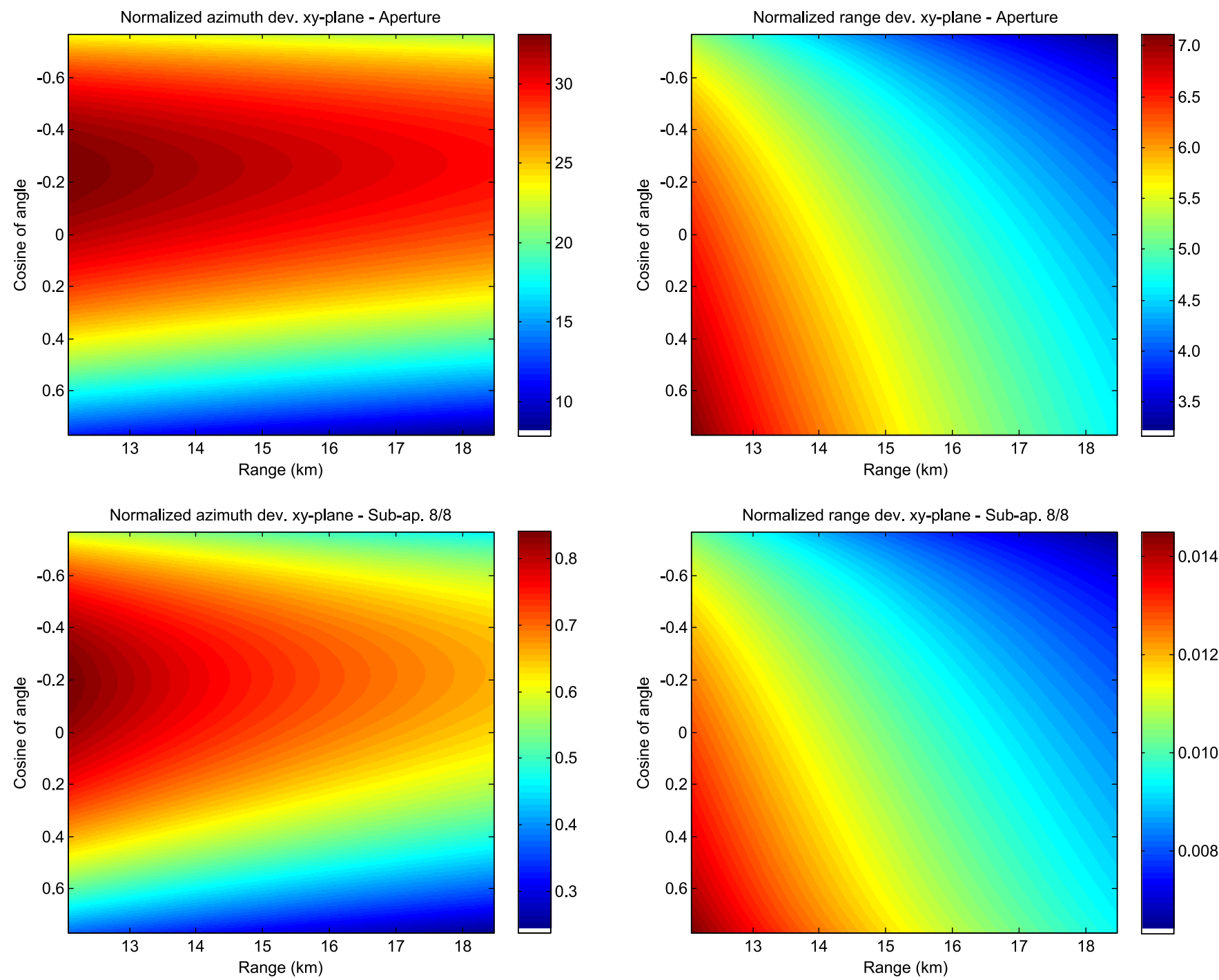

Fig. 6. Sensitivity plots for the second data set (Linköping), corresponding to a scenario where an erroneous $x y z$-acceleration $\left(a_{x}=+0.0025 \mathrm{~m} / \mathrm{s}^{2}, a_{y}=\right.$ $-0.0025 \mathrm{~m} / \mathrm{s}^{2}$, and $a_{z}=+0.0025 \mathrm{~m} / \mathrm{s}^{2}$ ) has been superposed on the measured flight track. (Top, left) $\varepsilon_{\alpha}$-plot for the final factorization step. (Top, right) $\varepsilon_{\rho}$-plot for the final factorization step. (Bottom, left to right) Worst-case sensitivity plots, three factorization steps back. Note that the top plots have values well above unity, whereas the bottom plots have values below.

\section{Data}

The FGA algorithm with the new search strategy incorporated, has been applied on real CARABAS II [14] data (two different scenes: Vidsel [30] and Linköping) acquired in stripmap mode. However, as radar echoes from a limited number of sample positions are factorized, the integration angle (and the angular resolution) varies as in spotlight mode.

The first data set (Vidsel) originates from a rural scene with a forest region and a lake. A few buildings, a trihedral reflector, and a power-line structure are prominent targets.

The second data set (Linköping) originates from an urban scene full of buildings and other man-made objects.

Table I resumes data-related information (system and geometry quantities).

\section{Realization and Evaluation}

To motivate the use of autofocus, errors due to a constant acceleration $\left(a_{x}=+0.0025 \mathrm{~m} / \mathrm{s}^{2}, a_{y}=-0.0025 \mathrm{~m} / \mathrm{s}^{2}, a_{z}=\right.$ $+0.0025 \mathrm{~m} / \mathrm{s}^{2}$ ) are superposed on the measured flight track
TABLE II

Number of Parameters USED AT THE Three FGA STEPS, I.E., BOTH FOR THE LOW-ORDER SEARCH (STAGE 1) AND FOR THE Local-TO-GLobal COMPUTATION (STAGE 2)

\begin{tabular}{l|llll}
\hline Nr. of parameters & \multicolumn{2}{|l}{ Stage 1 (Blocks) } & & Stage 2 (full image) \\
\hline FGA step 1 & 1 & $L_{13}$ & 1 & $L_{13}$ \\
FGA step 2 & 1 & $L_{13}$ & 2 & $L_{13}$ and $\Delta L$ \\
FGA step 3 & 2 & $L_{13}$ and $\Delta L$ & 3 & $L_{13}, \Delta L$ and $v$ \\
\hline
\end{tabular}

(verified to be measured accurately, i.e., from a focus perspective). To a first order, this simulates the drift of a stand-alone IMU. To be more specific, the chosen acceleration corresponds to the bias of a typical intermediate grade IMU [13], often used in smaller aircrafts and helicopters.

The sensitivity analysis is used to compare the measured track and the false track. $\varepsilon_{\alpha^{-}}$and $\varepsilon_{\rho}$-plots are shown in Figs. 5 and 6, i.e., for Vidsel and Linköping respectively.

The plots at the top correspond to the final factorization step. As shown, the focus criterion is violated, i.e., $\varepsilon_{\alpha}$ and $\varepsilon_{\rho}$ exceed unity. Hence, it should be obvious that, if autofocus is omitted 

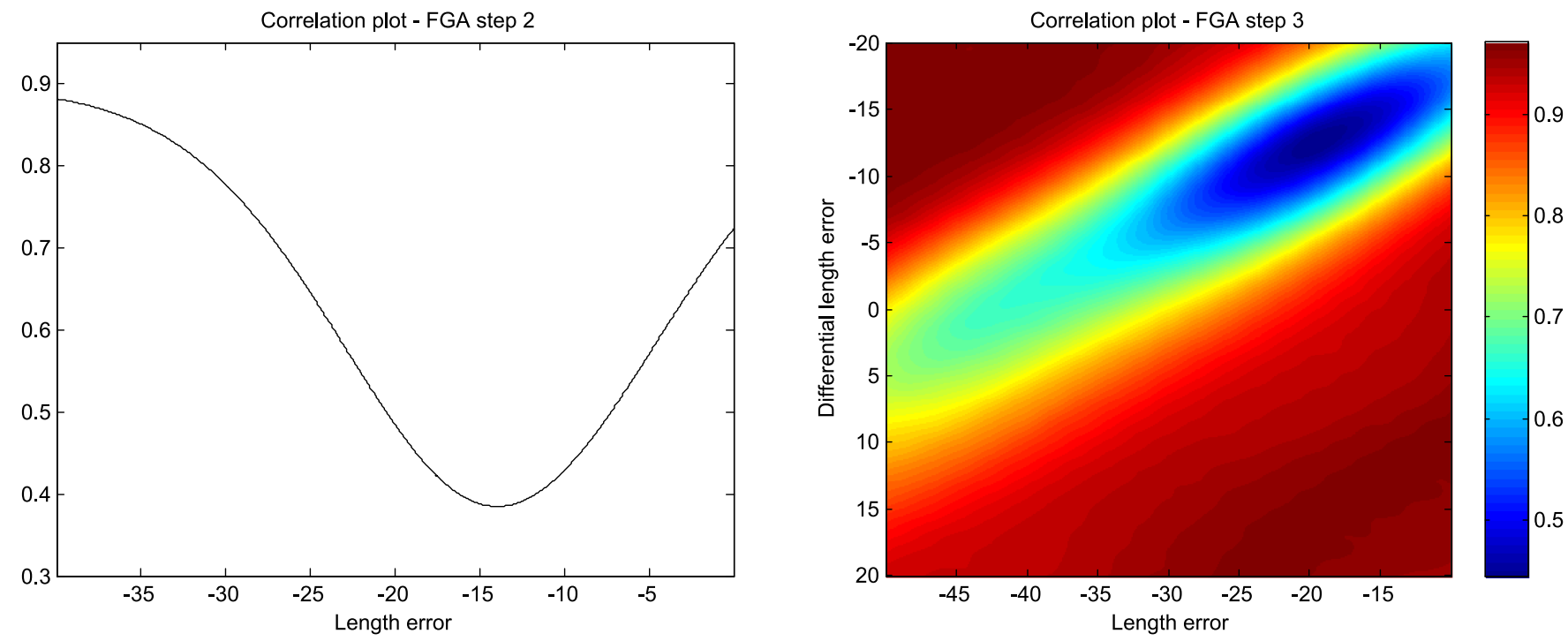

Fig. 7. Correlation plots for a block (Vidsel). (Left) Typical correlation plot at the second FGA step; one parameter $\left(L_{13}\right)$ does the job. (Right) Correlation plot at the third and final FGA step; note that two parameters $\left(L_{13}\right.$ and $\left.\Delta L\right)$ are required at this stage, i.e., to perform a low-order search. A plot for the first step is omitted as it would look like the one to the left.
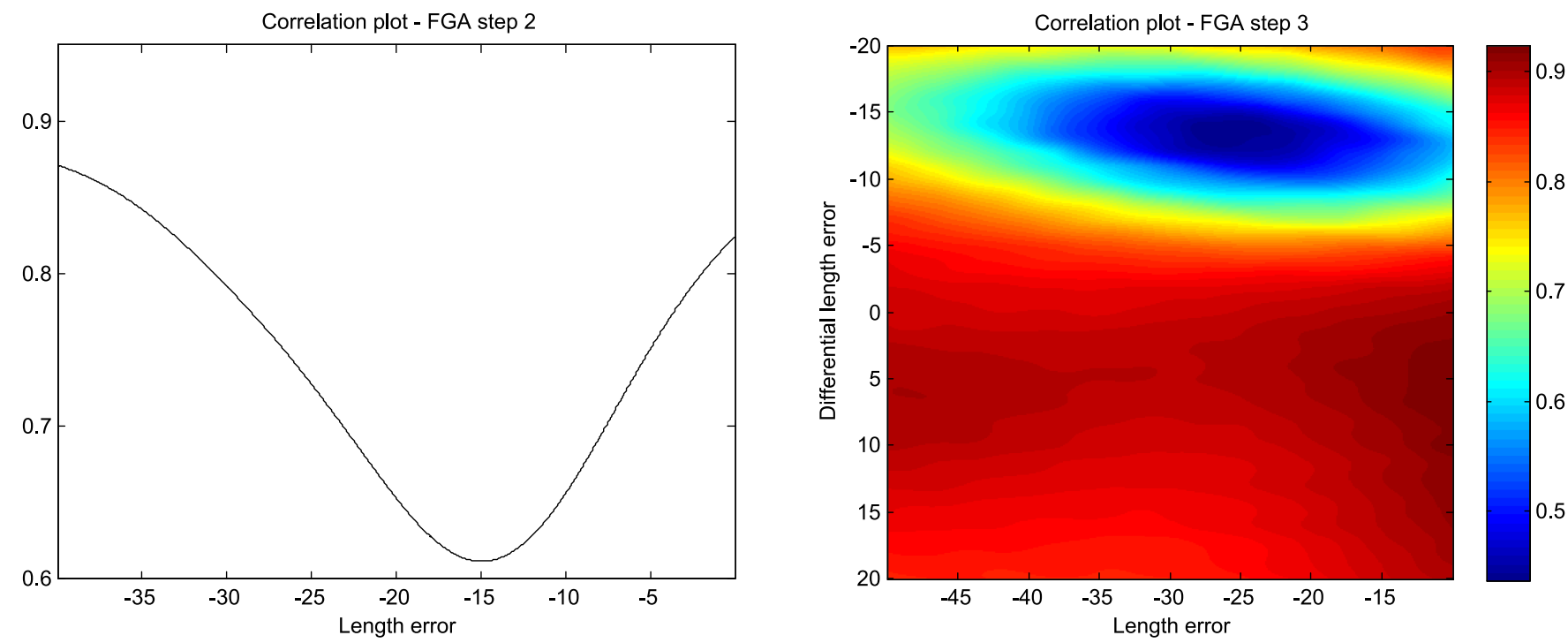

Fig. 8. Correlation plots for a block (Linköping). (Left) Typical correlation plot at the second FGA step; one parameter $\left(L_{13}\right)$ does the job. (Right) Correlation plot at the third and final FGA step; note that two parameters $\left(L_{13}\right.$ and $\left.\Delta L\right)$ are required at this stage, i.e., to perform a low-order search. A plot for the first step is omitted as it would look like the one to the left.

in the processing chain, the final image will be defocused. For example, when $\varepsilon_{\alpha}$-values differ by more than two normalized units across the plot, defocusing effects will be space variant. Very high $\varepsilon_{\alpha}$-values (greater than $\sim 10$ normalized units) in turn indicate the presence of residual RCM. Similar conclusions can be drawn based on $\varepsilon_{\rho}$-values.

The plots at the bottom correspond to the factorization step where 16 subapertures are merged into eight. Note that there are eight pairs of plots available at this step. However, only the worst-case pair is shown. As this is the last factorization step satisfying the focus criteria, the sensitivity analysis states that three FGA steps are required to compensate for the errors.

Now, before executing the FGA algorithm, eight subimages are formed by way of GBP, i.e., by employing (1) with limits: $n=1 \rightarrow N / 8 ; N / 8+1 \rightarrow N / 4$, etc. (i.e., the aperture (the track) is partitioned into adjacent subapertures). Complex
$D\left(n, R_{n}\right)$ values are found through nearest neighbor interpolation of discrete radar echoes, upsampled eight times in slant range (giving a sample spacing about 36 times finer than Nyquist, due to initial upsampling) via zero padding in the (range) frequency domain. Note that defocusing effects are not significant up until now.

The FGA algorithm is then executed in three steps, i.e., subapertures are factorized in three steps until an aperture image is obtained. For this purpose, 2-D cubic spline [15] interpolation is adopted. Naturally, the geometry is regulated by means of the new search strategy. One parameter $\left(L_{13}\right)$ should be set at the first step, two parameters $\left(L_{13}\right.$ and $\left.\Delta L\right)$ suffice at the second step, and three parameters $\left(L_{13}, \Delta L\right.$, and $\left.v\right)$ must be adjusted at the third step (once again this information is deduced from the sensitivity analysis). Note that these quantities are involved in the local-to-global computation (stage 2). The low-order stage 

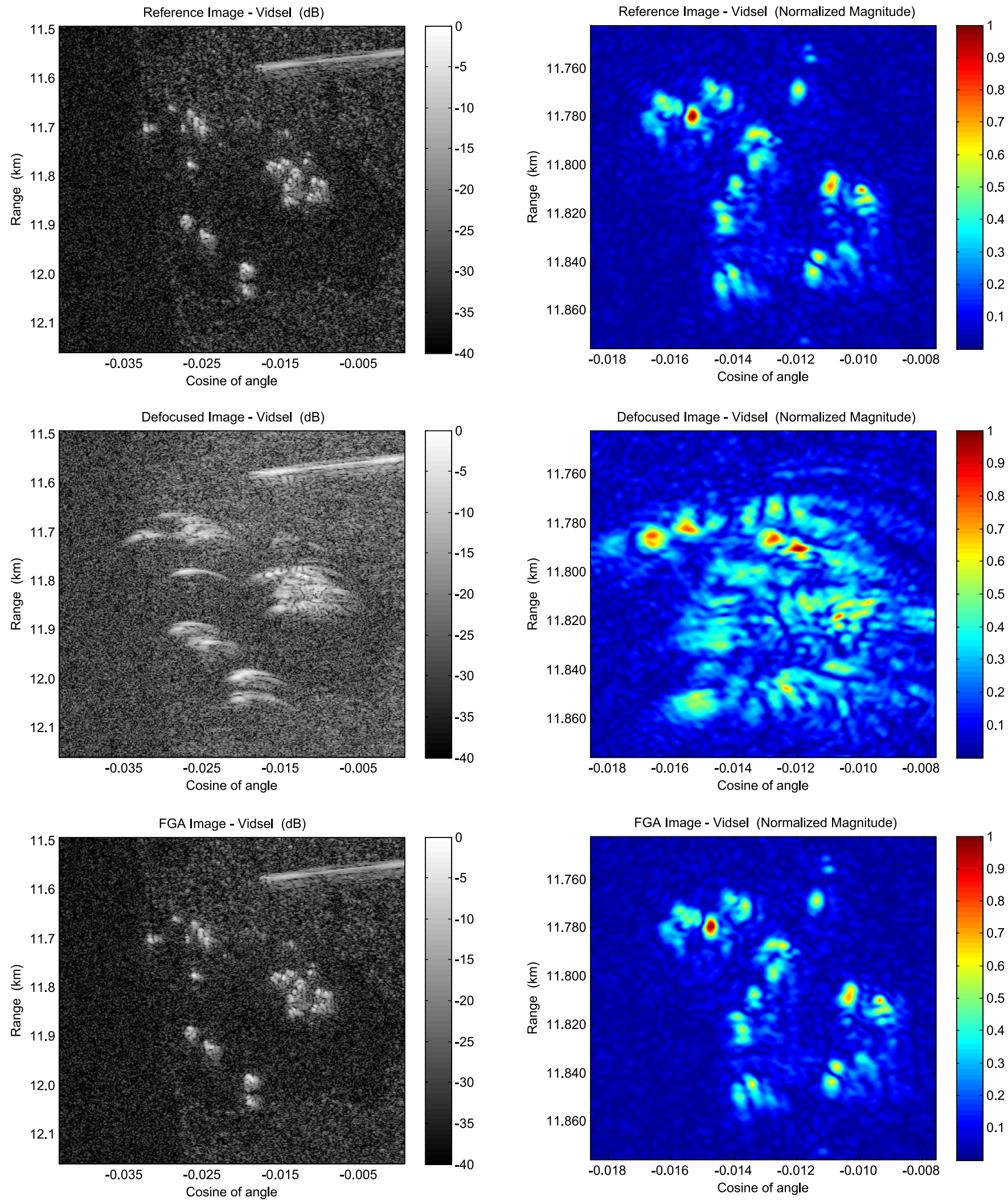

Fig. 9. (Left, top to bottom) Reference (focused) image, defocused image, and autofocused (FGA) image for the first data set (Vidsel). The defocused image displays residual RCM and SNR degradation. The FGA image is in turn very similar (basically identical) to the reference image. (Right, top to bottom) Closeup views in corresponding left-side images. Magnitude and color scale have been chosen over decibel and grayscale to accentuate details better when looking closer.

(stage 1) adopts the same or fewer parameters, i.e., one quantity $\left(L_{13}\right)$ for the first two steps and two $\left(L_{13}\right.$ and $\left.\Delta L\right)$ for the third (see Table II for a complete summary). In this paper, images are partitioned into 25 blocks. Correlation plots for one block, illustrating the object function $(F)$, are shown in Figs. 7 and 8, i.e., for Vidsel and Linköping, respectively. 

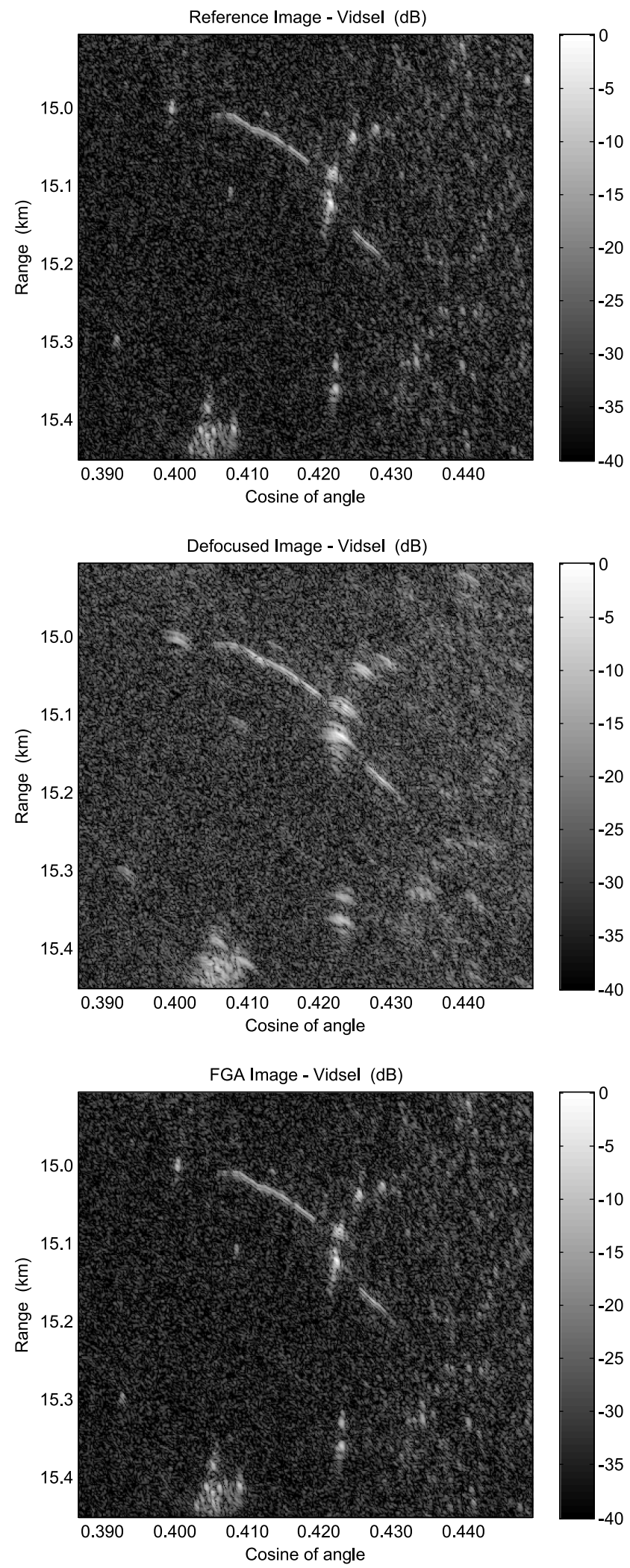
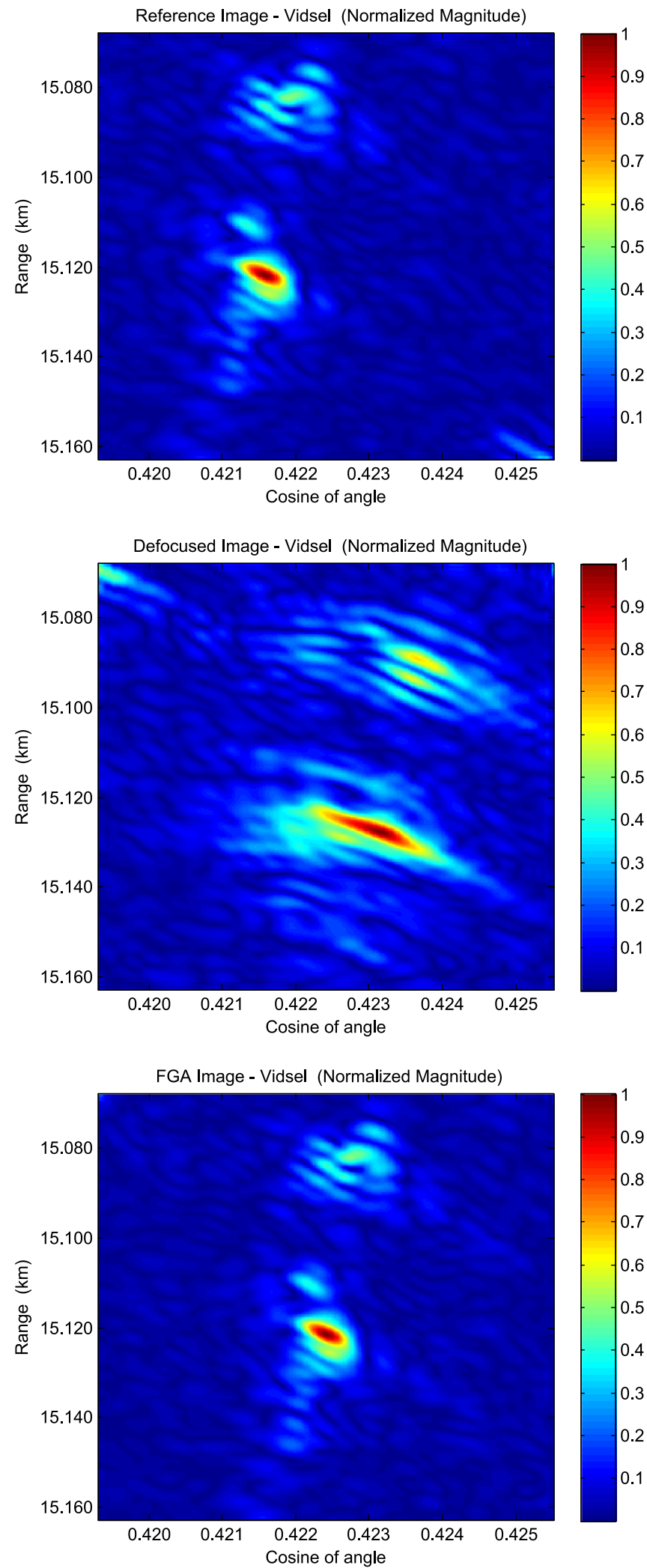

Fig. 10. (Left, top to bottom) Reference (focused) image, defocused image, and autofocused (FGA) image. The FGA image and the reference image essentially look the same. (Right, top to bottom) Closeup views in corresponding left-side images. Note that the FGA image has been translated horizontally (a nondefocusing effect).

After the (final) FGA image has been formed, a point-like target is extracted. The image chip is then upsampled via zero padding in the 2-D frequency domain, before taking resolution, peak-tosidelobe ratio (PSLR), and peak magnitude measurements.
Apart from an FGA image, a reference image (no autofocus, no errors superposed) and a defocused image (no autofocus, errors superposed) are also produced. The complete collection enables a comprehensive comparison. 

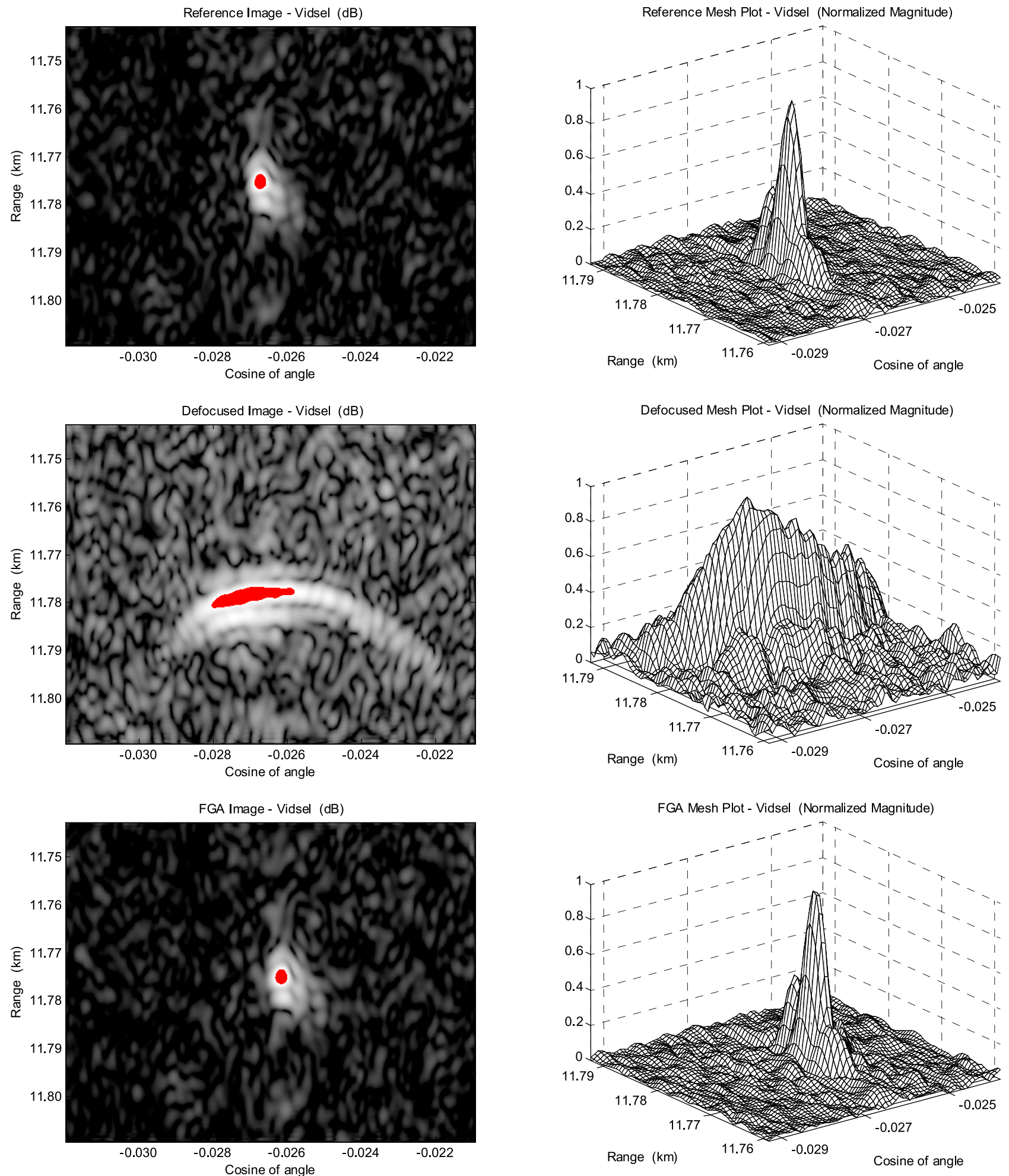

Fig. 11. (Left, top to bottom) Image chips for a point-like target (trihedral); 3-dB areas are marked red. (Right, top to bottom) Corresponding 3-D mesh plots. See also Fig. 9.

\section{RESULTS}

Results are presented here (see also Figs. 9-14).

Aperture images for the first data set, i.e., Vidsel, can be studied in Figs. 9 and 10. Note that these images are parts of one large image, formed for the scene size stated in Table I. The parts are offset in azimuth and range with respect to one an- other; this fulfills the criterion for space variance (see previous section and sensitivity plots in Fig. 5).

It is clear that defocusing effects are compensated by the FGA algorithm. Apart from just observing the visual similarity between FGA and reference images, this is confirmed through measurements on a point-like target, i.e., a trihedral reflector 

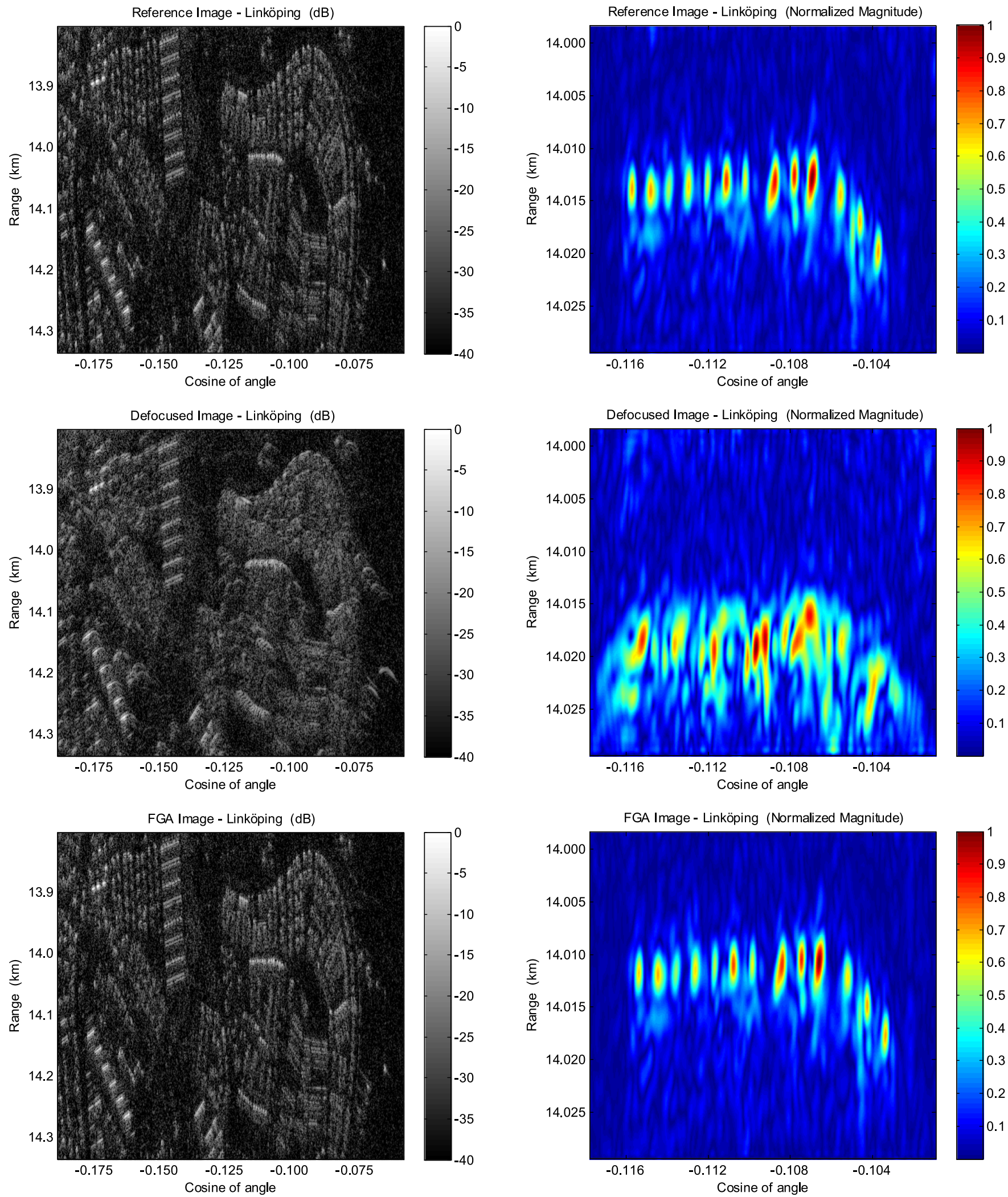

Fig. 12. (Left, top to bottom) Reference (focused) image, defocused image, and autofocused (FGA) image for the second data set (Linköping). The defocused image displays residual RCM and SNR degradation. The FGA image is in turn very similar (basically identical) to the reference image. (Right, top to bottom) Closeup views in corresponding left-side images. Magnitude and color scale have been chosen over decibel and grayscale to accentuate details better when looking closer.

(see Fig. 11). Before measuring, the target is upsampled 50 times by way of zero padding in the 2-D frequency domain.

With respect to the reference image, the width of the main lobe in the FGA image is within $\sim 1 \%$ in both azimuth and range. The PSLR loss is negligible (less than $0.1 \mathrm{~dB}$ ), whereas the peak magnitude loss is about $0.2 \mathrm{~dB}$.

Aperture images for the second data set, i.e., Linköping, can be studied in Figs. 12 and 13. These parts are also offset in 

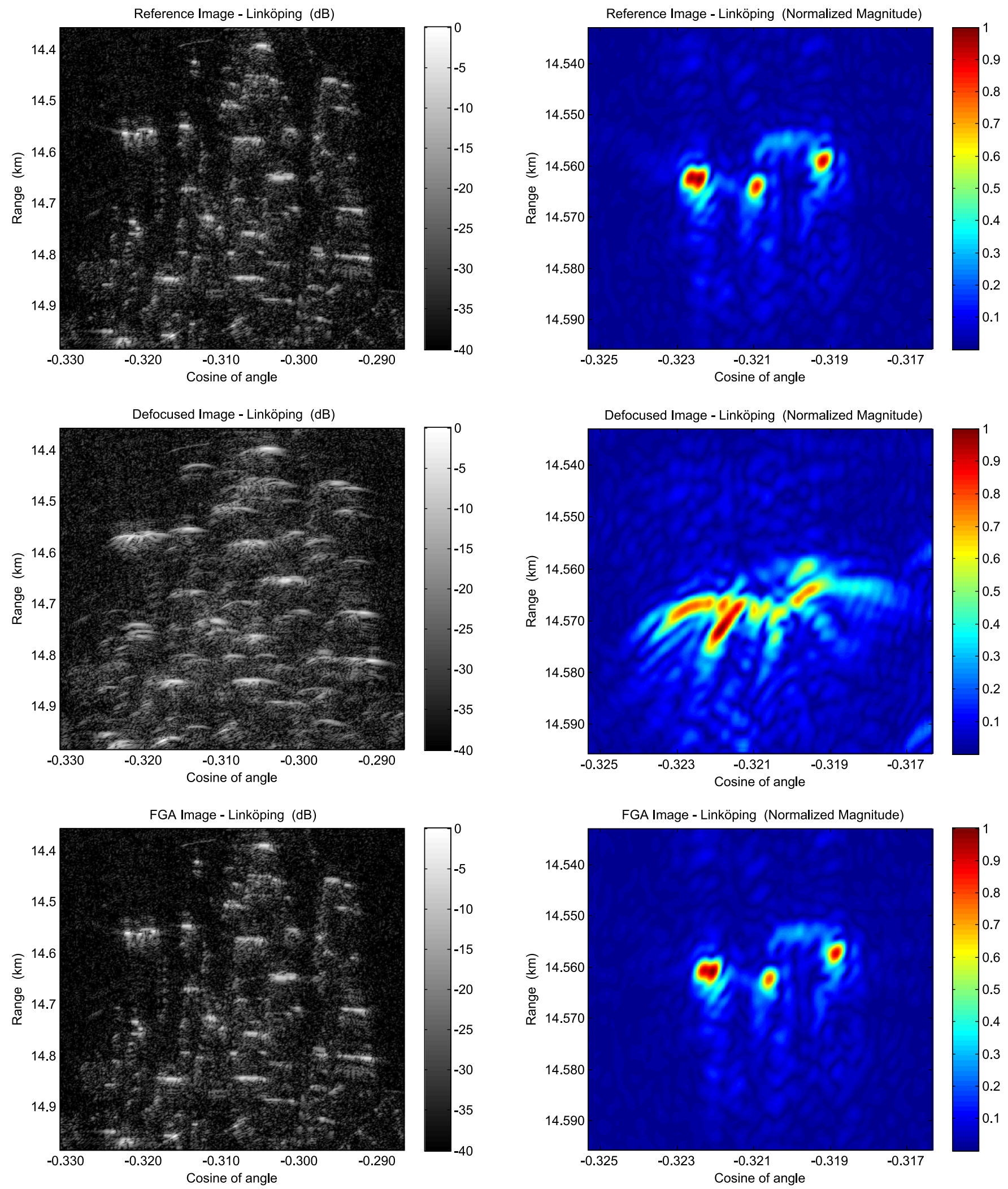

Fig. 13. (Left, top to bottom) Reference (focused) image, defocused image, and autofocused (FGA) image. The FGA image and the reference image essentially look the same. (Right, top to bottom) Closeup views in corresponding left-side images.

azimuth and range to fulfill the criterion for space variance (see previous section and sensitivity plots in Fig. 6).

Once again, a clear visual similarity between FGA and reference images can be observed. Measurements on a point-like target (see Fig. 14) confirm this further. The same upsampling factor as before is adopted.

With respect to the reference image, the width of the main lobe in the FGA image is within $\sim 1 \%$ in both azimuth and range. 

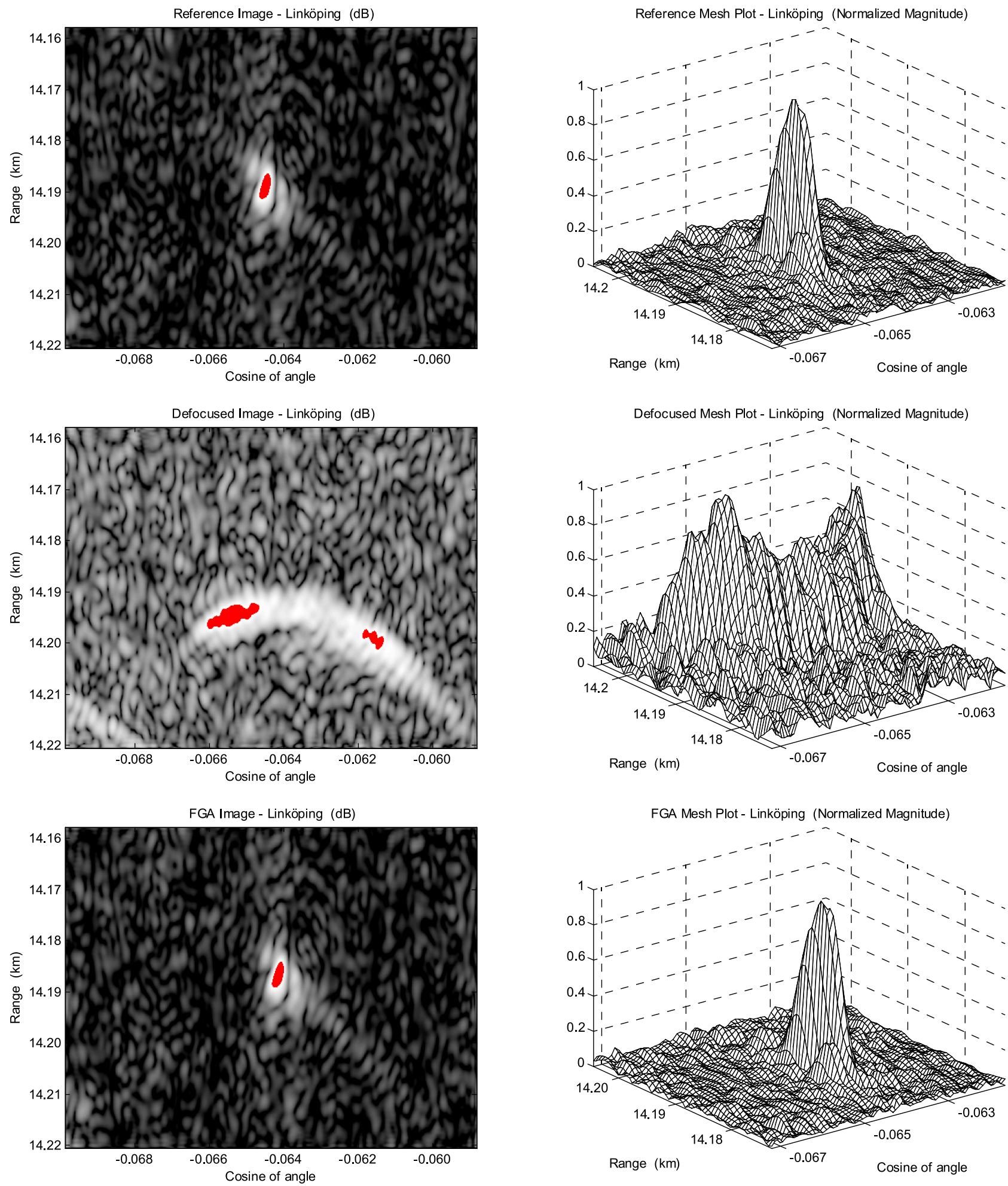

Fig. 14. (Left, top to bottom) Image chips for a point-like target; 3-dB areas are marked red. (Right, top to bottom) Corresponding 3-D mesh plots. See also Fig. 12.

The PSLR loss is approximately $0.4 \mathrm{~dB}$, whereas the peak magnitude loss is about $0.2 \mathrm{~dB}$.

Applied and estimated error quantities are not close. At the third FGA step, the estimated $L_{13}$ error for Vidsel is for example more than $10 \mathrm{~m}$ off. The estimated $v$ error for Linköping is in turn negative instead of positive. As a reduced parameter set has been adopted, this does not come as a surprise. Despite large geometrical differences, FGA images are still focused.
This implies that there are multiple solutions to the autofocus problem. The original flight track is one. The dominant effect of the geometrical changes is a $\sim 5 \mathrm{~m}$ image translation in azimuth (for Vidsel and Linköping). For Linköping, the image is also translated $\sim 2 \mathrm{~m}$ in the range direction orthogonal to azimuth. Deviations from pure translations are limited to $\sim 0.5 \mathrm{~m}$ and $\sim 1.5 \mathrm{~m}$ for Vidsel and Linköping, respectively, i.e., across the scenes. 
In essence, image results are satisfying. The FGA algorithm corrects residual space-variant RCM, indicating that the search strategy performs well.

\section{Discussion}

Results have now been presented (see Figs. 9-14). It is evident that focused (reference) and autofocused (FGA) images are very similar. Target measurements also verify this.

Hence, the search strategy locates a suitable geometry, i.e., one out of several.

In principle, numerous different solutions will produce a sharp image. Small variations of less sensitive parameters $(\phi$, $\beta_{13}$, and $H_{13}$ ) will for example not affect target measurements appreciably. For the first two FGA steps, $v$ can be adjusted instead of $L_{13}$. The sensitivity analysis confirms this for the first search stage and for the second stage. By adopting more parameters than required for this autofocus problem, the good geometry solutions increase in number. This has been verified by evaluating other geometries as well. However, adding extra parameters is a risky approach, as the number of poor solutions also will increase. In our case, only the minima are of interest [see (21)]. All suitable geometries do not satisfy this condition. In addition, some of the geometries corresponding to minima will produce a defocused image. Strictly speaking, whether there is an absolute minimum for (21) that is satisfying from a focus perspective is not known. Further studies are required.

Note also that only defocusing effects are compensated; therefore, the image may be translated, rotated, and distorted. In principle this implies that, if geometrical precision is crucial, a postprocessing stage may be required to correct nondefocusing effects. This would involve cross-referencing known targets in the image to a map, solving an equation system, and ultimately resampling.

The local-to-global computation is time efficient (executed in a few seconds), regardless of how many parameters the global solution calls for. A few trials actually achieve faster convergence when more parameters are adopted. The low-order stage should still be streamlined, to be able to utilize the FGA algorithm for real-time applications. Subjects to consider in the context of execution efficiency are: number of blocks, the size of these, and of course interpolation accuracy.

The object function used by the FGA algorithm on each image area is also a topic for the future. Although the correlation based expression in (11) has served well thus far, there are numerous other functions to survey, e.g., contrast [9], [19], squared intensity [1], [8], [22], [25], entropy [8], [24], [32], etc. (note that these functions are applied on the sum in (10) and not on the terms. In [25], an optimal (sharpness) object function is derived based on the maximum likelihood theory and maximum posterior theory.

\section{CONCLUSION}

The FGA algorithm is a base-two FFBP realization where each subaperture pair, i.e., at each factorization step, comes with six adjustable geometry parameters. An image defocused due to (geometrical) measurement errors can be compensated by tuning these.
In this paper, we describe and assess a new search strategy within the scope of the FGA algorithm. The partially parallel two-stage approach first seeks to find low-order solutions or geometry hypotheses for a number of small image areas, i.e., by utilizing the FGA algorithm with as few parameters as possible (from a focus perspective). To autofocus the full image, a timeefficient local-to-global computation adapts a final geometry by making it similar to all individual hypotheses. Radar data can be dropped at this stage as calculations only involve elementary mathematical functions.

To demonstrate the performance, the FGA algorithm with the new search strategy incorporated is applied on real CARABAS II data. Acceleration errors are superposed on the measured flight track prior to processing, i.e., to set up a 6-D autofocus problem.

FGA and reference images are visually very similar. In addition, resolution, PSLR, and peak magnitude measurements on targets in corresponding images, give the same values within one percent and tenths of a decibel. These results also confirm the prediction of several satisfying autofocus solutions.

\section{ACKNOWLEDGMENT}

The authors would like to thank Dr. A. Åhlander, J. Lindgren (SAAB Electronic Defence Systems), and Dr. L. Eriksson (Chalmers University of Technology) for their valuable inputs. The authors would also like to thank the anonymous reviewers for their comments, helping to improve this paper.

\section{REFERENCES}

[1] J. N. Ash, "An autofocus method for backprojection imagery in synthetic aperture radar," IEEE Geosci. Remote Sens. Lett., vol. 9, no. 1, pp. 104-108, Jan. 2012.

[2] H. Cantalloube and P. Dubois-Fernandez, "Airborne X-band SAR imaging with $10 \mathrm{~cm}$ resolution: Technical challenge and preliminary results," Proc. Inst. Elect. Eng.-Radar, Sonar Navigat., vol. 153, no. 2, pp. 163-176, Apr. 2006.

[3] H. M. J. Cantalloube and C. E. Nahum, "Multiscale local map-drift-driven multilateration SAR autofocus using fast polar format image synthesis," IEEE Trans. Geosci. Remote Sens., vol. 49, no. 10, pp. 3730-3736, Oct. 2011.

[4] W. G. Carrara, R. S. Goodman, and R. M. Majewski, Spotlight Synthetic Aperture Radar: Signal Processing Algorithms. Norwood, MA, USA: Artech House, 1995.

[5] A. W. Doerry, "Autofocus correction of SAR images exhibiting excessive residual migration," in Proc. SPIE Radar Sens. Technol. IX, 2005, vol. 5788, pp. 34-45.

[6] P. H. Eichel, D. C. Ghiglia, and C. V. Jakowatz, "Speckle processing method for synthetic-aperture-radar phase correction," Opt. Lett., vol. 14, no. 20, pp. 1-3, Jan. 1989.

[7] P. H. Eichel and C. V. Jakowatz, "Phase-gradient algorithm as an optimal estimator of the phase derivative," Opt. Lett., vol. 14, no. 20, pp. 1101-1103, Oct. 1989.

[8] J. R. Fienup and J. J. Miller, "Aberration correction by maximizing generalized sharpness metrics," J. Opt. Soc., vol. 20, no. 4, pp. 609-620, 2003.

[9] P.-O. Frölind, A. Gustavsson, M. Lundberg, and L. M. H. Ulander, "Circular-aperture VHF-band synthetic aperture radar for detection of vehicles in forest concealment," IEEE Trans. Geosci. Remote Sens., vol. 50, no. 4, pp. 1329-1339, Apr. 2012.

[10] P.-O. Frölind and L. M. H. Ulander, "Evaluation of angular interpolation kernels in fast factorized backprojection," Proc. Inst. Elect. Eng.-Radar, Sonar Navigat., vol. 153, no. 3, pp. 243-249, Jun. 2006.

[11] Y. Gao, W. Yu, Y. Liu, R. Wang, and C. Shi, "Sharpness-based autofocusing for stripmap SAR using an adaptive-order polynomial model," IEEE Geosci. Remote Sens. Lett., vol. 11, no. 6, pp. 1086-1090, Jun. 2014.

[12] D. C. Ghiglia and G. A. Mastin, "Two-dimensional phase correction of synthetic-aperture-radar imagery," Opt. Lett., vol. 14, no. 20, pp. 1104-1106, Oct. 1989. 
[13] P. D. Groves, "Navigation using inertial sensors," IEEE Aerosp. Electron. Syst. Mag., vol. 30, no. 2, pp. 42-69, Feb. 2015.

[14] H. Hellsten, L. M. H. Ulander, A. Gustavsson, and B. Larsson, "Development of VHF CARABAS II SAR," in Proc. SPIE Radar Sens. Technol., 1996, vol. 2747, pp. 48-60.

[15] H. S. Hou and H. C. Andrews, "Cubic splines for image interpolation and digital filtering," IEEE Trans. Acoust., Speech, Signal Process., vol. 26, no. 6, pp. 508-517, Dec. 1978.

[16] C. V. Jakowatz and D. E. Wahl, "Eigenvector method for maximumlikelihood estimation of phase errors in synthetic-aperture-radar imagery," Opt. Lett., vol. 10, no. 12, pp. 2539-2546, 1993.

[17] C. V. Jakowatz, D. E. Wahl, P. H. Eichel, D. C. Ghiglia, and P. A. Thompson, Spotlight-Mode Synthetic Aperture Radar: A Signal Processing Approach. Norwell, MA, USA: Kluwer, 1996.

[18] J. C. Kirk, "Signal Based Motion Compensation (SBMC)," in Proc. IEEE Int. Radar Conf., 2000, pp. 463-468.

[19] J. Kolman, "PACE: An autofocus algorithm for SAR," in Proc. IEEE Int. Radar Conf., 2005, pp. 310-314.

[20] Y. Li, C. Liu, Y. Wang, and Q. Wang, “A robust motion error estimation method based on raw data," IEEE Trans. Geosci. Remote Sens., vol. 50, no. 7, pp. 2780-2790, Jul. 2012.

[21] J. R. Moreira, "A new method of aircraft motion error extraction from radar raw data for real time motion compensation," IEEE Trans. Geosci. Remote Sens., vol. 28, no. 4, pp. 620-626, Jul. 1990.

[22] R. L. Morrison, M. N. Do, and D. C. Munson, "SAR image autofocus by sharpness optimization: A theoretical study," IEEE Trans. Image Process. vol. 16, no. 9, pp. 2309-2321, Sep. 2007.

[23] J. Nocedal and S. J. Wright, Numerical Optimization. New York, NY, USA: Springer-Verlag, 2006.

[24] B. D. Rigling, "Multistage entropy minimization for SAR image autofocus," in Proc. SPIE Algorithms Synthetic Aperture Radar Imagery XII, 2006, vol. 6237, pp. 1-10.

[25] T. J. Schulz, "Optimal sharpness function for SAR autofocus," IEEE Signal Process. Lett., vol. 14, no. 1, pp. 27-30, Jan. 2007.

[26] J. Torgrimsson, P. Dammert, H. Hellsten, and L. M. H. Ulander, "Factorized geometrical autofocus for synthetic aperture radar processing," IEEE Trans. Geosci. Remote Sens., vol. 52, no. 10, pp. 6674-6687, Oct. 2014.

[27] J. Torgrimsson, P. Dammert, H. Hellsten, and L. M. H. Ulander, "Autofocus and analysis of geometrical errors within the framework of fast factorized back-projection," in Proc. SPIE Algorithms Synthetic Aperture Radar Imagery XXI, 2014, vol. 9093, pp. 1-16.

[28] L. M. H. Ulander and P.-O. Frölind, "Ultra-wideband SAR interferometry," IEEE Trans. Geosci. Remote Sens., vol. 36, no. 5, pp. 1540-1550, Sep. 1998.

[29] L. M. H. Ulander, H. Hellsten, and G. Stenström, "Synthetic aperture radar processing using fast factorized backprojection," IEEE Trans. Aerosp. Electron. Syst., vol. 39, no. 3, pp. 760-776, Jul. 2003.

[30] L. M. H. Ulander, M. Lundberg, W. Pierson, and A. Gustavsson, "Change detection for low-frequency SAR ground surveillance," Proc. Inst. Elect. Eng.-Radar, Sonar Navigat., vol. 152, no. 6, pp. 413-420, Dec. 2005.

[31] M. Xing, X. Jiang, R. Wu, F. Zhou, and Z. Bao, "Motion compensation for UAV SAR based on raw radar data," IEEE Trans. Aerosp. Electron. Syst., vol. 47, no. 8, pp. 2870-2883, Aug. 2009.

[32] A. F. Yegulalp, "Minimum entropy SAR autofocus," presented at the ASAP Workshop, Lexington, MA, USA, 1999.

[33] D. Zhu, R. Jiang, X. Mao, and Z. Zhu, "Multi-subaperture PGA for SAR autofocusing," IEEE Trans. Aerosp. Electron. Syst., vol. 49, no. 1, pp. 468-488, Jan. 2013.

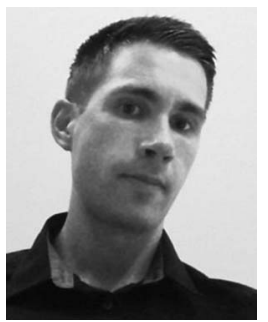

Jan Torgrimsson received the M.Sc. degree in engineering electronics from Umeå University, Umeå, Sweden, in 2009 and the Ph.D. degree in synthetic aperture radar autofocus from Chalmers University of Technology, Gothenburg, Sweden, in 2015.

$\mathrm{He}$ is currently a Postdoctoral Researcher with the Radar Remote Sensing Group, at Chalmers University of Technology. His main research interest is synthetic-aperture-radar processing, with emphasis on image formation algorithms and autofocus.

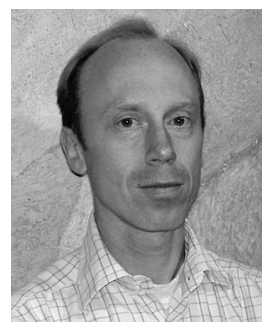

Patrik Dammert (S'95-M'00-SM'07) received the M.Sc. degree in electrical engineering and the Ph.D. degree in the applications of spaceborne SAR interferometry from the Chalmers University of Technology, Gothenburg, Sweden, in 1993 and 1999, respectively.

From 1999 to 2000, he worked as a Research Assistant with the Chalmers University of Technology, until joining Saab Electronic Defence Systems (formerly Ericsson Microwave Systems), Gothenburg. At Saab, he has been responsible for the development of high-performance SAR systems for airborne radars, spanning from very high frequency band to X-band systems (with flat plate antennas and with active electronically scanned array antennas). He has also been a Project Manager and an Associate Doctoral Student Supervisor for research projects at Saab in collaborations with the Chalmers University of Technology and the Blekinge Institute of Technology, Karlskrona, Sweden. His research interests include SAR systems, radar modeling, algorithms and signal processing, autofocus, and target detection in heavy-tailed radar clutter.

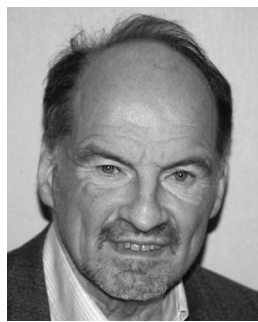

Hans Hellsten (M'06) received the Ph.D. degree in theoretical physics from Stockholm University, Stockholm, Sweden, in 1981.

$\mathrm{He}$ worked as a Research Director with the Swedish Defence Research Agency (FOI). He is currently a Senior Radar Expert with Saab Electronic Defence Systems, Gothenburg, Sweden. He is also the Head of the low-frequency radar product segment at Saab. He is, since the start of low-frequency radar development in 1985, the originator and one of the main developers of this technology, first at FOI, and since 2001, at Saab. In 1987, together with Lars-Erik Andersson, he formulated and solved for the first time the exact synthetic aperture radar reconstruction problem. Later on, he codeveloped with Lars Ulander the factorized back projection method as practical realization of the exact solution. He is the author or coauthor of numerous scientific and technical publications. He is the inventor of about 30 patents, granted in Sweden and other countries, mainly as the sole inventor.

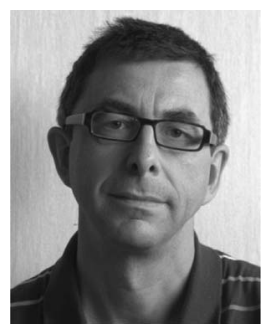

Lars M. H. Ulander (S'86-M'90-SM'04) received the M.Sc. degree in engineering physics and the Ph.D. degree in electrical and computer engineering from Chalmers University of Technology, Gothenburg, Sweden, in 1985 and 1991, respectively.

Since 1995, he has been with the Swedish Defence Research Agency (FOI), where he is the Director of Research in Radar Signal Processing and leads the research on very high frequency/ultrahigh frequency band radar. Since 2014, he has been a Professor in radar remote sensing with Chalmers University of Technology. He is the author or coauthor of over 300 professional publications, of which more than 60 are in peer-reviewed scientific journals. He is the holder of five patents. His research interests include synthetic aperture radar, electromagnetic scattering models, and remote sensing applications. 